\title{
COMPUTING $\alpha$-INVARIANTS OF SINGULAR DEL PEZZO SURFACES
}

\author{
IVAN CHELTSOV AND DIMITRA KOSTA
}

\begin{abstract}
We prove new local inequality for divisors on surfaces and utilize it to compute $\alpha$-invariants of singular del Pezzo surfaces, which implies that del Pezzo surfaces of degree one whose singular points are of type $\mathbb{A}_{1}, \mathbb{A}_{2}, \mathbb{A}_{3}, \mathbb{A}_{4}, \mathbb{A}_{5}$ or $\mathbb{A}_{6}$ are Kähler-Einstein.
\end{abstract}

We assume that all varieties are projective, normal, and defined over $\mathbb{C}$.

\section{INTRODUCTION}

Let $X$ be a Fano variety with at most quotient singularities (a Fano orbifold).

Theorem 1.1 ([37]). If $\operatorname{dim}(X)=2$ and $X$ is smooth, then

the surface $X$ is Kähler-Einstein $\Longleftrightarrow$ the group $\operatorname{Aut}(X)$ is reductive.

An important role in the proof of Theorem 1.1 is played by several holomorphic invariants, which are now known as $\alpha$-invariants. Let us describe their algebraic counterparts.

Let $D$ be an effective $\mathbb{Q}$-divisor on the variety $X$. Then the number

$$
\mathrm{c}(X, D)=\sup \{\epsilon \in \mathbb{Q} \mid \text { the } \log \text { pair }(X, \epsilon D) \text { is } \log \text { canonical }\} \in \mathbb{Q} \cup\{+\infty\} .
$$

is called the log canonical threshold of the divisor $D$ (see [21, Definition 8.1]). Put

$$
\operatorname{lct}_{n}(X)=\inf \left\{\mathrm{c}\left(X, \frac{1}{n} B\right) \mid B \text { is a divisor in }\left|-n K_{X}\right|\right\}
$$

for every $n \in \mathbb{N}$. For small $n$, the number $\operatorname{lct}_{n}(X)$ is usually not very hard to compute.

Example $1.2([28])$. If $X$ is a smooth surface in $\mathbb{P}^{3}$ of degree 3 , then

$$
\operatorname{lct}_{1}(X)=\left\{\begin{array}{l}
2 / 3 \text { if } X \text { has an Eckardt point, } \\
3 / 4 \text { if } X \text { has no Eckardt points. }
\end{array}\right.
$$

The number $\operatorname{lct}_{n}(X)$ is denoted by $\alpha_{n}(X)$ in 38 .

Remark 1.3. It follows from [27, Lemma 4.8] that the set

$$
\left\{\mathrm{c}\left(X, \frac{1}{n} B\right) \mid B \text { is a divisor in }\left|-n K_{X}\right|\right\}
$$

is finite (cf. [23]). Thus, there exists a divisor $B \in\left|-n K_{X}\right| \operatorname{such}_{\text {that }} \operatorname{lct}_{n}(X)=\mathrm{c}(X, B / n) \in \mathbb{Q}$.

If the variety $X$ is smooth, then it is proved by Demailly (see [․ Theorem A.3]) that

$$
\inf \left\{\operatorname{lct}_{n}(X) \mid n \in \mathbb{N}\right\}=\alpha(X)
$$

where $\alpha(X)$ is the $\alpha$-invariant introduced by Tian in [36]. Put $\operatorname{lct}(X)=\inf \left\{\operatorname{lct}_{n}(X) \mid n \in \mathbb{N}\right\}$.

Conjecture 1.4 ([38, Question 1]). There is an $n \in \mathbb{N}$ such that $\operatorname{lct}(X)=\operatorname{lct}_{n}(X)$.

The authors thank G. Brown, N. Budur, J. Kollár, M. Mustata, J. Park, Y. Prokhorov for valuable comments.

The authors would like to thank an anonymous referee for many useful remarks.

This paper was completed under financial support provided by IKY (Greek State Scholarship Foundation). 
The proof of Theorem 1.1 uses (at least implicitly) the following result.

Theorem 1.5 ([36, [10]). The Fano orbifold $X$ is Kähler-Einstein if

$$
\operatorname{lct}(X)>\frac{\operatorname{dim}(X)}{\operatorname{dim}(X)+1} .
$$

Note that there are many well-known obstructions to the existence of Kähler-Einstein metrics on smooth Fano manifolds and Fano orbifolds (see [25], [14, [15], 34]).

Example 1.6. If $X \cong \mathbb{P}(1,2,3)$, then $X$ is not Kähler-Einstein (see [15], 34]).

Let us describe one more $\alpha$-invariant that took its origin in [37].

Let $\mathcal{M}$ be a linear system on the variety $X$. Then the number

$$
\mathrm{c}(X, \mathcal{M})=\sup \{\epsilon \in \mathbb{Q} \mid \text { the log pair }(X, \epsilon \mathcal{M}) \text { is } \log \text { canonical }\} \in \mathbb{Q} \cup\{+\infty\} .
$$

is called the $\log$ canonical threshold of the linear system $\mathcal{M}$ (cf. [21, Theorem 4.8]). Put

$$
\operatorname{lct}_{n, 2}(X)=\inf \left\{\mathrm{c}\left(X, \frac{1}{n} \mathcal{B}\right) \mid \mathcal{B} \text { is a pencil in }\left|-n K_{X}\right|\right\}
$$

for every $n \in \mathbb{N}$. The number $\operatorname{lct}_{n, 2}(X)$ is denoted by $\alpha_{n, 2}(X)$ in [8] and [4]. Note that

$$
\operatorname{lct}(X)=\inf \left\{\operatorname{lct}_{n, 2}(X) \mid n \in \mathbb{N}\right\},
$$

and it follows from [21, Theorem 4.8] that $\operatorname{lct}_{n}(X) \leqslant \operatorname{lct}_{n, 2}(X)$ for every $n \in \mathbb{N}$.

Remark 1.8. It follows from [27, Lemma 4.8] and [21, Theorem 4.8] that the set

$$
\left\{\mathrm{c}\left(X, \frac{1}{n} \mathcal{B}\right) \mid \mathcal{B} \text { is a pencil in }\left|-n K_{X}\right|\right\}
$$

is finite. Thus, there is a pencil $\mathcal{B}$ in $\left|-n K_{X}\right|$ such that the equality $\operatorname{lct}_{n, 2}(X)=\mathrm{c}(X, \mathcal{B} / n)$. Then

$$
\operatorname{lct}_{n, 2}(X)>\operatorname{lct}(X)
$$

if there exists at most finitely many effective $\mathbb{Q}$-divisors $D_{1}, D_{2}, \ldots, D_{r}$ on the variety $X$ such that

$$
\mathrm{c}\left(X, D_{1}\right)=\mathrm{c}\left(X, D_{2}\right)=\cdots=\mathrm{c}\left(X, D_{r}\right)=\operatorname{lct}(X)
$$

and $D_{1} \sim_{\mathbb{Q}} D_{2} \sim_{\mathbb{Q}} \ldots \sim_{\mathbb{Q}} D_{r} \sim_{\mathbb{Q}}-K_{X}$.

The importance of the number $\operatorname{lct}_{n, 2}(X)$ is due to the following conjecture.

Conjecture 1.9 (cf. [8, Theorem 2], [41, Theorem 1]). Suppose that

$$
\operatorname{lct}_{n, 2}(X)>\frac{\operatorname{dim}(X)}{\operatorname{dim}(X)+1} \text {. }
$$

for every $n \in \mathbb{N}$. Then $X$ is Kähler-Einstein.

Note that Conjecture 1.9 is not much stronger than Theorem 1.5 by (1.7).

Example 1.10. Suppose that $X$ is a smooth hypersurface in $\mathbb{P}^{m}$ of degree $m \geqslant 3$. Then

$$
\operatorname{lct}_{n}(X) \geqslant 1-\frac{1}{m}=\frac{\operatorname{dim}(X)}{\operatorname{dim}(X)+1}
$$

for every $n \in \mathbb{N}$ by [2]. The equality $\operatorname{lct}_{n}(X)=1-1 / m$ holds $\Longleftrightarrow$ the hypersurface $X$ contains a cone of dimension $m-2$ (see [2, Theorem 1.3], [2, Theorem 4.1], [13, Theorem 0.2]). Then

$$
\operatorname{lct}_{n, 2}(X)>\frac{\operatorname{dim}(X)}{\operatorname{dim}(X)+1}
$$


by Remark 1.8, [2, Remark 1.6], [2, Theorem 4.1], [2, Theorem 5.2] and [13, Theorem 0.2], because $X$ contains at most finitely many cones by [9, Theorem 4.2]. If $X$ is general, then

$$
1=\operatorname{lct}_{1}(X) \geqslant \operatorname{lct}(X) \geqslant\left\{\begin{array}{l}
3 / 4 \text { if } m=3, \\
7 / 9 \text { if } m=4, \\
5 / 6 \text { if } m=5, \\
1 \text { if } m \geqslant 5,
\end{array}\right.
$$

by [33], 3], [5]. Thus, if $X$ is general, then it is Kähler-Eisntein by Theorem 1.5.

The assertion of Conjecture 1.9 follows from [8, Theorem 2] and [41, Theorem 1] under an additional assumption that the Kähler-Ricci flow on $X$ is tamed (see [8] and [41]).

Theorem 1.11 ([8], [41]). If $\operatorname{dim}(X)=2$, then the Kähler-Ricci flow on $X$ is tamed.

Corollary 1.12. Suppose that $\operatorname{dim}(X)=2$ and

$$
\operatorname{lct}_{n, 2}(X)>\frac{2}{3}
$$

for every $n \in \mathbb{N}$. Then $X$ is Kähler-Einstein.

Two-dimensional Fano orbifolds are called del Pezzo surfaces.

Remark 1.13. Del Pezzo surfaces with quotient singularities are not classified (cf. [20]). But

- del Pezzo surfaces with canonical singularities are classified (see [18]),

- del Pezzo surfaces with 2-Gorenstein quotient singularities are classified (see [1]),

- del Pezzo surfaces of Picard rank 1 with $T$-singularities are classified (see [17]).

Del Pezzo surfaces with canonical singularities form a very natural class of del Pezzo surfaces.

Problem 1.14. Describe all Kähler-Einstein del Pezzo surface with canonical singularities.

Recall that if $X$ is a del Pezzo surface with canonical singularities, then

- either the inequality $K_{X}^{2} \geqslant 5$ holds,

- or one of the following possible cases occurs:

- the equality $K_{X}^{2}=1$ holds and $X$ is a sextic surface in $\mathbb{P}(1,1,2,3)$,

- the equality $K_{X}^{2}=2$ holds and $X$ is a quartic surface in $\mathbb{P}(1,1,1,2)$,

- the equality $K_{X}^{2}=3$ holds and $X$ is a cubic surface in $\mathbb{P}^{3}$,

- the equality $K_{X}^{2}=4$ holds and $X$ is a complete intersection in $\mathbb{P}^{4}$ of two quadrics.

Let us consider few examples to illustrate the expected answer to Problem 1.14.

Example 1.15. Suppose that $X$ is a sextic surface in $\mathbb{P}(1,1,2,3)$ such that its singular locus consists of singular points of type $\mathbb{A}_{1}$ or $\mathbb{A}_{2}$. Arguing as in the proof of [3, Lemma 4.1], we see that

$$
\operatorname{lct}_{n, 2}(X)>\frac{2}{3}
$$

for every $n \in \mathbb{N}$. Thus, the surface $X$ is Kähler-Einstein by Corollary 1.12 ,

Example 1.16. Suppose that $X$ is a quartic surface in $\mathbb{P}(1,1,1,2)$ such that its singular locus consists of singular points of type $\mathbb{A}_{1}$ or $\mathbb{A}_{2}$. Then $X$ is Kähler-Einstein by [16, Theorem 2].

Example 1.17. Suppose that $X$ is a cubic surface in $\mathbb{P}^{3}$ that is not a cone. Then

- if $X$ is smooth, then $X$ is Kähler-Einstein by Theorem 1.1.

- if $\operatorname{Sing}(X)$ consists of one point of type $\mathbb{A}_{1}$, then it follows from [35, Theorem 5.1] that

$$
\operatorname{lct}_{n, 2}(X)>\frac{2}{3}=\operatorname{lct}_{1}(X)=\operatorname{lct}(X)
$$

for every $n \in \mathbb{N}$, which implies that $X$ is Kähler-Einstein by Corollary 1.12 , 
- if the cubic surface $X$ has a singular point that is not a singular point of type $\mathbb{A}_{1}$ or $\mathbb{A}_{2}$, then the surface $X$ is not Kähler-Einstein by [11, Proposition 4.2].

Example 1.18. Suppose that $X$ is a complete intersection in $\mathbb{P}^{4}$ of two quadrics. Then

- if $X$ is smooth, then $X$ is Kähler-Einstein by Theorem 1.1,

- if $X$ is Kähler-Einstein, then $X$ has at most singular points of type $\mathbb{A}_{1}$ (see [19]),

- it follows from [24] or [16, Theorem 44] that $X$ is Kähler-Einstein if it is given by

$$
\sum_{i=0}^{4} x_{i}^{2}=\sum_{i=0}^{4} \lambda_{i} x_{i}^{2}=0 \subseteq \mathbb{P}^{4} \cong \operatorname{Proj}\left(\mathbb{C}\left[x_{0}, \ldots, x_{4}\right]\right),
$$

and $X$ has at most singular points of type $\mathbb{A}_{1}$, where $\left(\lambda_{0}: \lambda_{1}: \lambda_{2}: \lambda_{3}: \lambda_{4}\right) \in \mathbb{P}^{4}$.

Keeping in mind Examples 1.15, 1.16, 1.17 and 1.18, [4, Example 1.12] and [26, Table 1], it is very natural to expect that the following answer to Problem 1.14 is true (cf. Example 1.6).

Conjecture 1.19. If the orbifold $X$ is a del Pezzo surface with at most canonical singularities, then the surface $X$ is Kähler-Enstein $\Longleftrightarrow$ it satisfies one of the following conditions:

- $K_{X}^{2}=1$ and $\operatorname{Sing}(X)$ consists of points of type $\mathbb{A}_{1}, \mathbb{A}_{2}, \mathbb{A}_{3}, \mathbb{A}_{4}, \mathbb{A}_{5}, \mathbb{A}_{6}, \mathbb{A}_{7}$ or $\mathbb{D}_{4}$,

- $K_{X}^{2}=2$ and $\operatorname{Sing}(X)$ consists of points of type $\mathbb{A}_{1}, \mathbb{A}_{2}$ or $\mathbb{A}_{3}$,

- $K_{X}^{2}=3$ and $\operatorname{Sing}(X)$ consists of points of type $\mathbb{A}_{1}$ or $\mathbb{A}_{2}$,

- $K_{X}^{2}=4$ and $\operatorname{Sing}(X)$ consists of points of type $\mathbb{A}_{1}$,

- the surface $X$ is smooth and $6 \geqslant K_{X}^{2} \geqslant 5$,

- either $X \cong \mathbb{P}^{2}$ or $X \cong \mathbb{P}^{1} \times \mathbb{P}^{1}$.

In this paper, we prove the following result.

Theorem 1.20. Suppose that $X$ is a sextic surface in $\mathbb{P}(1,1,2,3)$. Then

$$
\operatorname{lct}_{n, 2}(X)>\frac{2}{3}
$$

for every $n \in \mathbb{N}$ if $\operatorname{Sing}(X)$ consists of points of type $\mathbb{A}_{1}, \mathbb{A}_{2}, \mathbb{A}_{3}, \mathbb{A}_{4}, \mathbb{A}_{5}$ or $\mathbb{A}_{6}$.

Corollary 1.21. Suppose that $X$ is a sextic surface in $\mathbb{P}(1,1,2,3)$ such that its singular locus consists of singular points of type $\mathbb{A}_{1}, \mathbb{A}_{2}, \mathbb{A}_{3}, \mathbb{A}_{4}, \mathbb{A}_{5}$ or $\mathbb{A}_{6}$. Then $X$ is Kähler-Enstein.

It should be pointed out that Corollary 1.21 and Examples 1.15, 1.16, 1.17, 1.18 illustrate a general philosophy that the existence of Kähler-Enstein metrics on Fano orbifolds is related to an algebro-geometric notion of stability (see [11, Theorem 4.1], [39], [12]).

Remark 1.22. If $X$ is a sextic surface in $\mathbb{P}(1,1,2,3)$ with canonical singularities, then either

$$
\operatorname{Sing}(X) \in\left\{\begin{array}{l}
\mathbb{E}_{8}, \mathbb{E}_{7}, \mathbb{E}_{7}+\mathbb{A}_{1}, \mathbb{E}_{6}, \mathbb{E}_{6}+\mathbb{A}_{2}, \mathbb{E}_{6}+\mathbb{A}_{1}, \mathbb{D}_{8}, \mathbb{D}_{7}, \mathbb{D}_{6}, \mathbb{D}_{6}+\mathbb{A}_{1}+\mathbb{A}_{1}, \mathbb{D}_{6}+\mathbb{A}_{1}, \\
\mathbb{D}_{5}, \mathbb{D}_{5}+\mathbb{A}_{3}, \mathbb{D}_{5}+\mathbb{A}_{2}, \mathbb{D}_{5}+\mathbb{A}_{1}+\mathbb{A}_{1}, \mathbb{D}_{5}+\mathbb{A}_{1}, \mathbb{D}_{4}, \mathbb{D}_{4}+\mathbb{D}_{4}, \mathbb{D}_{4}+\mathbb{A}_{3}, \mathbb{D}_{4}+\mathbb{A}_{2}, \\
\mathbb{D}_{4}+\mathbb{A}_{1}+\mathbb{A}_{1}+\mathbb{A}_{1}+\mathbb{A}_{1}, \mathbb{D}_{4}+\mathbb{A}_{1}+\mathbb{A}_{1}+\mathbb{A}_{1}, \mathbb{D}_{4}+\mathbb{A}_{1}+\mathbb{A}_{1}, \mathbb{D}_{4}+\mathbb{A}_{1}, \mathbb{A}_{8}, \\
\mathbb{A}_{7}, \mathbb{A}_{7}+\mathbb{A}_{1}, \mathbb{A}_{6}, \mathbb{A}_{6}+\mathbb{A}_{1}, \mathbb{A}_{5}, \mathbb{A}_{5}+\mathbb{A}_{1}, \mathbb{A}_{5}+\mathbb{A}_{1}+\mathbb{A}_{1}, \mathbb{A}_{5}+\mathbb{A}_{2}, \mathbb{A}_{5}+\mathbb{A}_{2}+\mathbb{A}_{1}, \\
\mathbb{A}_{4}, \mathbb{A}_{4}+\mathbb{A}_{4}, \mathbb{A}_{4}+\mathbb{A}_{3}, \mathbb{A}_{4}+\mathbb{A}_{2}+\mathbb{A}_{1}, \mathbb{A}_{4}+\mathbb{A}_{2}, \mathbb{A}_{4}+\mathbb{A}_{1}+\mathbb{A}_{1}, \mathbb{A}_{4}+\mathbb{A}_{1}, \\
\mathbb{A}_{3}, \mathbb{A}_{3}+\mathbb{A}_{3}, \mathbb{A}_{3}+\mathbb{A}_{3}+\mathbb{A}_{1}+\mathbb{A}_{1}, \mathbb{A}_{3}+\mathbb{A}_{2}, \mathbb{A}_{3}+\mathbb{A}_{2}+\mathbb{A}_{1}, \mathbb{A}_{3}+\mathbb{A}_{2}+\mathbb{A}_{1}+\mathbb{A}_{1}, \\
\mathbb{A}_{3}+\mathbb{A}_{1}+\mathbb{A}_{1}+\mathbb{A}_{1}+\mathbb{A}_{1}, \mathbb{A}_{3}+\mathbb{A}_{1}+\mathbb{A}_{1}+\mathbb{A}_{1}, \mathbb{A}_{3}+\mathbb{A}_{1}+\mathbb{A}_{1}, \mathbb{A}_{3}+\mathbb{A}_{1}
\end{array}\right\}
$$

or $\operatorname{Sing}(X)$ consists only of points of type $\mathbb{A}_{1}$ and $\mathbb{A}_{2}$ (see [40]).

What is known about $\alpha$-invariants of del Pezzo surfaces with canonical singularities?

Theorem $1.23([3])$. If $X$ is a smooth del Pezzo surface, then $\operatorname{lct}(X)=\operatorname{lct}_{1}(X)$. 
Theorem 1.24 ([3], [31]). If $X$ is a del Pezzo surface with canonical singularities, then

$$
\operatorname{lct}(X)=\operatorname{lct}_{1}(X)
$$

in the case when $K_{X}^{2} \geqslant 3$.

Theorem 1.25 ([31]). If $X$ is a quartic surface in $\mathbb{P}(1,1,1,2)$ with canonical singularities, then

$$
\operatorname{lct}(X)=\left\{\begin{array}{l}
\operatorname{lct}_{2}(X)=1 / 3 \text { if } X \text { has a singular point of type } \mathbb{A}_{7}, \\
\operatorname{lct}_{2}(X)=2 / 5 \text { if } X \text { has a singular point of type } \mathbb{A}_{6}, \\
\operatorname{lct}_{1}(X) \text { in the remaining cases. }
\end{array}\right.
$$

In this paper, we prove the following result (cf. Example 1.15).

Theorem 1.26. Suppose that $X$ is a sextic surface in $\mathbb{P}(1,1,2,3)$ with canonical singularities, let $\omega: X \rightarrow \mathbb{P}(1,1,2)$ be a natural double cover, and let $R$ be its branch curve in $\mathbb{P}(1,1,2)$. Then

$$
\operatorname{lct}(X)=\left\{\begin{array}{l}
\operatorname{lct}_{2}(X)=1 / 3 \text { if } \operatorname{Sing}(X) \text { consists of a point of type } \mathbb{D}_{8}, \\
\operatorname{lct}_{2}(X)=2 / 5 \text { if } \operatorname{Sing}(X) \text { consists of a point of type } \mathbb{D}_{7}, \\
\operatorname{lct}_{3}(X)=1 / 2 \text { if } \operatorname{Sing}(X) \text { consists of a point of type } \mathbb{A}_{8}, \\
\operatorname{lct}_{2}(X)=1 / 2 \text { if } \operatorname{Sing}(X) \text { consists of a point of type } \mathbb{A}_{7} \text { and a point of type } \mathbb{A}_{1}, \\
\operatorname{lct}_{2}(X)=1 / 2 \text { if } \operatorname{Sing}(X) \text { consists of a point of type } \mathbb{A}_{7} \text { and } R \text { is reducible, } \\
\operatorname{lct}_{3}(X)=3 / 5 \text { if } X \text { has a singular point of type } \mathbb{A}_{7} \text { and } R \text { is irreducible, } \\
\operatorname{lct}_{2}(X)=2 / 3 \text { if } X \text { has a singular point of type } \mathbb{A}_{6}, \\
\operatorname{lct}_{2}(X)=2 / 3 \text { if } X \text { has a singular point of type } \mathbb{A}_{5}, \\
\operatorname{lct}_{2}(X)=\min (\operatorname{lct}(X), 4 / 5) \text { if } X \text { has a singular point of type } \mathbb{A}_{4}, \\
\operatorname{lct}_{1}(X) \text { in the remaining cases. }
\end{array}\right.
$$

It should be pointed out that if $X$ is a del Pezzo surface with at most canonical singularities, then all possible values of the number $\operatorname{lct}_{1}(X)$ are computed in [28], 29], 30].

Example 1.27. If $X$ is a sextic surface in $\mathbb{P}(1,1,2,3)$ with canonical singularities, then

- $\operatorname{lct}_{1}(X)=1 / 6 \Longleftrightarrow$ the surface $X$ has a singular point of type $\mathbb{E}_{8}$,

- $\operatorname{lct}_{1}(X)=1 / 4 \Longleftrightarrow$ the surface $X$ has a singular point of type $\mathbb{E}_{7}$,

- $\operatorname{lct}_{1}(X)=1 / 3 \Longleftrightarrow$ the surface $X$ has a singular point of type $\mathbb{E}_{6}$,

- $\operatorname{lct}_{1}(X)=1 / 2 \Longleftrightarrow$ the surface $X$ has a singular point of type $\mathbb{D}_{4}, \mathbb{D}_{5}, \mathbb{D}_{6}, \mathbb{D}_{7}$ or $\mathbb{D}_{8}$,

- $\operatorname{lct}_{1}(X)=2 / 3 \Longleftrightarrow$ the following two conditions are satisfied:

- the surface $X$ has no singular points of type $\mathbb{D}_{4}, \mathbb{D}_{5}, \mathbb{D}_{6}, \mathbb{D}_{7}, \mathbb{D}_{8}, \mathbb{E}_{6}, \mathbb{E}_{7}$ or $\mathbb{E}_{8}$,

- there is a curve in $\left|-K_{X}\right|$ that has a cusp at a point in $\operatorname{Sing}(X)$ of type $\mathbb{A}_{2}$,

- $\operatorname{lct}_{1}(X)=3 / 4 \Longleftrightarrow$ the following three conditions are satisfied:

- the surface $X$ has no singular points of type $\mathbb{D}_{4}, \mathbb{D}_{5}, \mathbb{D}_{6}, \mathbb{D}_{7}, \mathbb{D}_{8}, \mathbb{E}_{6}, \mathbb{E}_{7}$ or $\mathbb{E}_{8}$,

- there is no curve in $\left|-K_{X}\right|$ that has a cusp at a point in $\operatorname{Sing}(X)$ of type $\mathbb{A}_{2}$,

- there is a curve in $\left|-K_{X}\right|$ that has a cusp at a point in $\operatorname{Sing}(X)$ of type $\mathbb{A}_{1}$,

- $\operatorname{lct}_{1}(X)=5 / 6 \Longleftrightarrow$ the following three conditions are satisfied:

- the surface $X$ has no singular points of type $\mathbb{D}_{4}, \mathbb{D}_{5}, \mathbb{D}_{6}, \mathbb{D}_{7}, \mathbb{D}_{8}, \mathbb{E}_{6}, \mathbb{E}_{7}$ or $\mathbb{E}_{8}$,

- there is no curve in $\left|-K_{X}\right|$ that have a cusp at a point in $\operatorname{Sing}(X)$,

- there is a curve in $\left|-K_{X}\right|$ that has a cusp,

- $\operatorname{lct}_{1}(X)=1 \Longleftrightarrow$ there are no cuspidal curves in $\left|-K_{X}\right|$.

A crucial role in the proofs of both Theorems 1.26 and 1.20 is played by a new local inequality that we discovered. This inequality is a technical tool, but let us describe it now. 
Let $S$ be a surface, let $D$ be an arbitrary effective $\mathbb{Q}$-divisor on the surface $S$, let $O$ be a smooth point of the surface $S$, let $\Delta_{1}$ and $\Delta_{2}$ be reduced irreducible curves on $S$ such that

$$
\Delta_{1} \not \operatorname{Supp}(D) \nsupseteq \Delta_{2},
$$

and the divisor $\Delta_{1}+\Delta_{2}$ has a simple normal crossing singularity at the smooth point $O \in \Delta_{1} \cap \Delta_{2}$, let $a_{1}$ and $a_{2}$ be some non-negative rational numbers. Suppose that the log pair

$$
\left(S, D+a_{1} \Delta_{1}+a_{2} \Delta_{2}\right)
$$

is not Kawamata log terminal at $O$, but $\left(S, D+a_{1} \Delta_{1}+a_{2} \Delta_{2}\right)$ is Kawamata log terminal in a punctured neighborhood of the point $O$.

Theorem 1.28. Let $A, B, M, N, \alpha, \beta$ be non-negative rational numbers. Then

$$
\operatorname{mult}_{O}\left(D \cdot \Delta_{1}\right) \geqslant M+A a_{1}-a_{2} \text { or } \operatorname{mult}_{O}\left(D \cdot \Delta_{2}\right) \geqslant N+B a_{2}-a_{1}
$$

in the case when the following conditions are satisfied:

- the inequality $\alpha a_{1}+\beta a_{2} \leqslant 1$ holds,

- the inequalities $A(B-1) \geqslant 1 \geqslant \max (M, N)$ hold,

- the inequalities $\alpha(A+M-1) \geqslant A^{2}(B+N-1) \beta$ and $\alpha(1-M)+A \beta \geqslant A$ hold,

- either the inequality $2 M+A N \leqslant 2$ holds or

$$
\alpha(B+1-M B-N)+\beta(A+1-A N-M) \geqslant A B-1 .
$$

Corollary 1.29. Suppose that

$$
\frac{2 m-2}{m+1} a_{1}+\frac{2}{m+1} a_{2} \leqslant 1
$$

for some integer $m$ such that $m \geqslant 3$. Then

$$
\operatorname{mult}_{O}\left(D \cdot \Delta_{1}\right) \geqslant 2 a_{1}-a_{2} \text { or } \operatorname{mult}_{O}\left(D \cdot \Delta_{2}\right) \geqslant \frac{m}{m-1} a_{2}-a_{1} .
$$

Proof. To prove the required assertion, let us put

$$
A=2, B=\frac{m}{m-1}, M=0, N=0, \alpha=\frac{2 m-2}{m+1}, \beta=\frac{2}{m+1} a_{2},
$$

and let us check that all hypotheses of Theorem 1.28 are satisfied.

We have $\alpha a_{1}+\beta a_{2} \leqslant 1$ by assumption. We have

$$
A(B-1)=\frac{2}{m-1} \geqslant 1 \geqslant 0=\max (M, N),
$$

since $m \geqslant 3$. We have

$$
\alpha(A+M-1)=\frac{2 m-2}{m+1} \geqslant \frac{8}{m^{2}-1}=A^{2}(B+N-1) \beta,
$$

since $m \geqslant 3$. We have $\alpha(1-M)+A \beta=2 \geqslant 2=A$ and $2 M+A N=0 \leqslant 2$.

Thus, we see that all hypotheses of Theorem 1.28 are satisfied. Then

$\operatorname{mult}_{O}\left(D \cdot \Delta_{1}\right) \geqslant M+A a_{1}-a_{2}=2 a_{1}-a_{2} \operatorname{or}_{\operatorname{mult}_{O}}\left(D \cdot \Delta_{2}\right) \geqslant N+B a_{2}-a_{1}=\frac{m}{m-1} a_{2}-a_{1}$

by Theorem 1.28 ,

For the convenience of a reader, we organize the paper in the following way:

- in Section 2, we collect auxiliary results,

- in Section 3, we prove Theorem 1.28,

- in Sections 4, we prove Theorem 4.1.

- in Sections 5, we prove Theorems 5.1.

- in Sections 6, we prove Theorems 6.1. 
By Remark 1.22, both Theorems 1.20 and 1.26 follow from Theorems 4.1 , 5.1 and 6.1 ,

\section{PReliminaries}

Let $S$ be a surface with canonical singularities, and let $D$ be an effective $\mathbb{Q}$-divisor on $S$. Put

$$
D=\sum_{i=1}^{r} a_{i} D_{i}
$$

where $D_{i}$ is an irreducible curve, and $a_{i} \in \mathbb{Q}_{>0}$. We assume that $D_{i} \neq D_{j} \Longleftrightarrow i \neq j$.

Suppose that $(S, D)$ is $\log$ canonical, but $(S, D)$ is not Kawamata log terminal.

Remark 2.1. Let $\bar{D}$ be an effective $\mathbb{Q}$-divisor on the surface $S$ such that

$$
\bar{D}=\sum_{i=1}^{r} \bar{a}_{i} D_{i} \sim_{\mathbb{Q}} D
$$

and the $\log$ pair $(S, \bar{D})$ is $\log$ canonical, where $\bar{a}_{i}$ is a non-negative rational number. Put

$$
\alpha=\min \left\{\frac{a_{i}}{\bar{a}_{i}} \mid \bar{a}_{i} \neq 0\right\}
$$

where $\alpha$ is well defined and $\alpha \leqslant 1$. Then $\alpha=1 \Longleftrightarrow D=\bar{D}$. Suppose that $D \neq \bar{D}$. Put

$$
D^{\prime}=\sum_{i=1}^{r} \frac{a_{i}-\alpha \bar{a}_{i}}{1-\alpha} D_{i}
$$

and choose $k \in\{1, \ldots, r\}$ such that $\alpha=a_{k} / \bar{a}_{k}$. Then $D_{k} \not \subset \operatorname{Supp}\left(D^{\prime}\right)$ and $D^{\prime} \sim_{\mathbb{Q}} \bar{D} \sim_{\mathbb{Q}} D$, but the log pair $\left(S, D^{\prime}\right)$ is not Kawamata log terminal.

Let $\operatorname{LCS}(S, D)$ be the locus of log canonical singularities of the log pair $(S, D)$ (see [6]).

Theorem 2.2 ([22, Theorem 17.4]). If $-\left(K_{S}+D\right)$ is nef and big, then $\operatorname{LCS}(S, D)$ is connected.

Take a point $P \in \operatorname{LCS}(S, D)$. Suppose that $\operatorname{LCS}(S, D)$ contains no curves that pass through $P$.

Lemma 2.3. Suppose that $P \notin \operatorname{Sing}(S)$ and $P \notin \operatorname{Sing}\left(D_{1}\right)$. Then

$$
D_{1} \cdot\left(\sum_{i=2}^{r} a_{i} D_{i}\right) \geqslant \sum_{i=2}^{r} a_{i} \operatorname{mult}_{P}\left(D_{1} \cdot D_{i}\right)>1 .
$$

Proof. The log pair $\left(S, D_{1}+\sum_{i=2}^{r} a_{i} D_{i}\right)$ is not log canonical at $P$, since $a_{1}<1$. Then

$$
D_{1} \cdot \sum_{i=2}^{r} a_{i} D_{i} \geqslant \sum_{i=2}^{r} a_{i} \operatorname{mult}_{P}\left(D_{1} \cdot D_{i}\right) \geqslant \operatorname{mult}_{P}\left(\left.\sum_{i=2}^{r} a_{i} D_{i}\right|_{D_{1}}\right)>1
$$

by [22, Theorem 17.6].

Let $\pi: \bar{S} \rightarrow S$ be a birational morphism, and $\bar{D}$ is a proper transform of $D$ via $\pi$. Then

$$
K_{\bar{S}}+\bar{D}+\sum_{i=1}^{s} e_{i} E_{i} \sim_{\mathbb{Q}} \pi^{*}\left(K_{S}+D\right),
$$

where $E_{i}$ is an irreducible $\pi$-exceptional curve, and $e_{i} \in \mathbb{Q}$. We assume that $E_{i}=E_{j} \Longleftrightarrow i=j$.

Suppose, in addition, that the birational morphism $\pi$ induces an isomorphism

$$
\bar{S} \backslash\left(\bigcup_{i=1}^{s} E_{i}\right) \cong S \backslash P .
$$

Remark 2.4. The log pair $\left(\bar{S}, \bar{D}+\sum_{i=1}^{s} e_{i} E_{i}\right)$ is not Kawamata log terminal at a point in $\cup_{i=1}^{s} E_{i}$. 
Suppose that $S$ is singular at $P$, and either $P$ is a singular point of type $\mathbb{D}_{n}$ for some $n \in \mathbb{N}_{\geqslant 4}$, or the point $P$ is a singular point of type $\mathbb{E}_{m}$ for some $m \in\{6,7,8\}$.

Lemma 2.5. Suppose that $E_{1}^{2}=E_{2}^{2}=\cdots=E_{s}^{2}=-2$. Then $e_{1}=1$ if

$$
E_{1} \cdot\left(\sum_{i=2}^{s} E_{i}\right)=3 .
$$

Proof. This follows from [32, Proposition 2.9], because $(S \ni P)$ is a weakly-exceptional singularity (see [32, Example 4.7], [7, Example 3.4], [7, Theorem 3.15]).

Lemma 2.6. Suppose that $S$ is a sextic surface in $\mathbb{P}(1,1,2,3)$ that has canonical singularities, and suppose that $D \sim_{\mathbb{Q}}-K_{X}$. Let $\mu$ be a positive rational number such that either

$$
\mu<\operatorname{lct}_{1}(S)
$$

or $\mu=2 / 3$ and $D$ is not a curve in $\left|-K_{X}\right|$ with a cusp at a point in $\operatorname{Sing}(S)$ of type $\mathbb{A}_{2}$. Then

$$
\operatorname{LCS}(S, \mu D) \subseteq \operatorname{Sing}(S),
$$

the locus $\operatorname{LCS}(S, \mu D)$ contains no points of type $\mathbb{A}_{1}$ or $\mathbb{A}_{2}$, and $|\operatorname{LCS}(S, \mu D)| \leqslant 1$.

Proof. This follows from Theorem 2.2 and the proof of [3, Lemma 4.1].

Most of the described results are valid in much more general settings (cf. [22] and [21]).

\section{LOCAL INEQUALITY}

The purpose of this section is to prove Theorem 1.28.

Let $S$ be a surface, let $D$ be an arbitrary effective $\mathbb{Q}$-divisor on the surface $S$, let $O$ be a smooth point of the surface $S$, let $\Delta_{1}$ and $\Delta_{2}$ be reduced irreducible curves on $S$ such that

$$
\Delta_{1} \not \subset \operatorname{Supp}(D) \nsupseteq \Delta_{2},
$$

and the divisor $\Delta_{1}+\Delta_{2}$ has a simple normal crossing singularity at the smooth point $O \in \Delta_{1} \cap \Delta_{2}$, let $a_{1}$ and $a_{2}$ be some non-negative rational numbers. Suppose that the log pair

$$
\left(S, D+a_{1} \Delta_{1}+a_{2} \Delta_{2}\right)
$$

is not Kawamata $\log$ terminal at $O$, but $\left(S, D+a_{1} \Delta_{1}+a_{2} \Delta_{2}\right)$ is Kawamata log terminal in a punctured neighborhood of the point $O$. In particular, we must have $a_{1}<1$ and $a_{2}<1$.

Let $A, B, M, N, \alpha, \beta$ be non-negative rational numbers such that

- the inequality $\alpha a_{1}+\beta a_{2} \leqslant 1$ holds,

- the inequalities $A(B-1) \geqslant 1 \geqslant \max (M, N)$ hold,

- the inequalities $\alpha(A+M-1) \geqslant A^{2}(B+N-1) \beta$ and $\alpha(1-M)+A \beta \geqslant A$ holds,

- either the inequality $2 M+A N \leqslant 2$ holds or

$$
\alpha(B+1-M B-N)+\beta(A+1-A N-M) \geqslant A B-1 .
$$

Lemma 3.1. The inequalities $A+M \geqslant 1$ and $B>1$ holds. The inequality

$$
\alpha(B+1-M B-N)+\beta(A+1-A N-M) \geqslant A B-1
$$

holds. The inequality $\beta(1-N)+B \alpha \geqslant B$ holds. The inequalities

$$
\frac{\alpha(2-M)}{A+1}+\frac{\beta(2-N)}{B+1} \geqslant 1
$$

and $\alpha(2-M) B+\beta(1-N)(A+1) \geqslant B(A+1)$ hold. 
Proof. The inequality $B>1$ follows from the inequality $A(B-1) \geqslant 1$. Then

$$
\frac{\alpha}{A+1}+\frac{\beta}{B+1} \geqslant \frac{\alpha}{A+1}+\frac{\beta}{2 B} \geqslant \frac{1}{2}
$$

because $2 B \geqslant B+1$. Similarly, we see that $A+M \geqslant 1$, because

$$
\frac{\alpha(A+M-1)}{A^{2}(B+N-1)} \geqslant \beta \geqslant 0
$$

and $B+N-1 \geqslant 0$. The inequality $\beta(1-N)+B \alpha \geqslant B$ follows from the inequalities

$$
\alpha+\frac{\beta(1-N)}{B} \geqslant \frac{2-M}{A+1} \alpha+\frac{\beta(1-N)}{B} \geqslant 1,
$$

because $A+1 \geqslant 2-M$.

Let us show that the inequality

$$
\alpha(2-M) B+\beta(1-N)(A+1) \geqslant B(A+1)
$$

holds. Let $L_{1}$ be the line in $\mathbb{R}^{2}$ given by the equation

$$
x(2-M) B+y(1-N)(A+1)-B(A+1)=0
$$

and let $L_{2}$ be the line that is given by the equation

$$
x(1-M)+A y-A=0,
$$

where $(x, y)$ are coordinates on $\mathbb{R}^{2}$. Then $L_{1}$ intersects the line $y=0$ at the point

$$
\left(\frac{A+1}{2-M}, 0\right)
$$

and $L_{2}$ intersects the line $y=0$ at the point $(A /(1-M), 0)$. But

$$
\frac{A+1}{2-M}<\frac{A}{1-M}
$$

which implies that $\alpha(2-M) B+\beta(1-N)(A+1) \geqslant B(A+1)$ if

$$
A^{2} \beta_{0}(B+N-1) \geqslant \alpha_{0}(A+M-1),
$$

where $\left(\alpha_{0}, \beta_{0}\right)$ is the intersection point of the lines $L_{1}$ and $L_{2}$. But

$$
\left(\alpha_{0}, \beta_{0}\right)=\left(\frac{A(A+1)(B+N-1)}{\Delta}, \frac{B(A-1+M)}{\Delta}\right),
$$

where $\Delta=2 A B-A B M-A+A M-1+M+N A-N A M+N-N M$. But

$$
A^{2}(B(A-1+M))(B+N-1) \geqslant(A(A+1)(B+N-1))(A+M-1),
$$

because $A(B-1) \geqslant 1$, which implies that $A^{2} \beta_{0}(B+N-1) \geqslant \alpha_{0}(A+M-1)$.

Finally, let us show that the inequality

$$
\alpha(B+1-M B-N)+\beta(A+1-A N-M) \geqslant A B-1
$$

holds. Let $L_{1}^{\prime}$ be the line in $\mathbb{R}^{2}$ given by the equation

$$
x(B+1-M B-N)+y \beta(A+1-A N-M)-A B+1=0
$$

where $(x, y)$ are coordinates on $\mathbb{R}^{2}$. Then $L_{1}^{\prime}$ intersects the line $y=0$ at the point

$$
\left(\frac{A B-1}{B+1-M B-N}, 0\right)
$$


and $L_{2}$ intersects the line $y=0$ at the point $(A /(1-M), 0)$. But

$$
\frac{A B-1}{B+1-M B-N}<\frac{A}{1-M}
$$

which implies that $\alpha(B+1-M B-N)+\beta(A+1-A N-M) \geqslant A B-1$ if

$$
A^{2} \beta_{1}(B+N-1) \geqslant \alpha_{1}(A+M-1),
$$

where $\left(\alpha_{1}, \beta_{1}\right)$ is the intersection point of the lines $L_{1}^{\prime}$ and $L_{2}$. Note that

$$
\left(\alpha_{1}, \beta_{1}\right)=\left(\frac{A(A B-A-2+N A+M)}{\Delta^{\prime}}, \frac{A+1-N A-M}{\Delta^{\prime}}\right),
$$

where $\Delta^{\prime}=A B-1-A B M+A M+2 M-N A M-M^{2}$.

To complete the proof, it is enough to show that the inequality

$$
A^{2}(A+1-N A-M)(B+N-1) \geqslant(A(A B-A-2+N A+M))(A+M-1)
$$

holds. This inequality is equivalent to the inequality

$$
(2-M)(A+M-1) \geqslant A(A N+2 M-2)(B+N-1),
$$

which is true, because $M \leqslant 1$ and $A N+2 M-2 \leqslant 0$.

Let us prove prove Theorem 1.28 by reductio ad absurdum. Suppose that the inequalities

$$
\operatorname{mult}_{O}\left(D \cdot \Delta_{1}\right)<M+A a_{1}-a_{2} \text { and } \operatorname{mult}_{O}\left(D \cdot \Delta_{2}\right)<N+B a_{2}-a_{1}
$$

hold. Let us show that this assumption leads to a contradiction.

Lemma 3.2. The inequalities $a_{1}>(1-M) / A$ and $a_{2}>(1-N) / B$ hold.

Proof. It follows from Lemma 2.3 that

$$
M+A a_{1}-a_{2}>\operatorname{mult}_{O}\left(D \cdot \Delta_{1}\right)>1-a_{2},
$$

which implies that $a_{1}>(1-M) / A$. Similarly, we see that $a_{2}>(1-N) / B$.

Put $m_{0}=\operatorname{mult}_{O}(D)$. Then $m_{0}$ is a positive rational number.

Remark 3.3. The inequalities $m_{0}<M+A a_{1}-a_{2}$ and $m_{0}<N+B a_{2}-a_{1}$ hold.

Lemma 3.4. The inequality $m_{0}+a_{1}+a_{2}<2$ holds.

Proof. We know that $m_{0}+a_{1}+a_{2}<M+(A+1) a_{1}$ and $m_{0}+a_{1}+a_{2}<N+(B+1) a_{2}$. Then

$$
\left(m_{0}+a_{1}+a_{2}\right)\left(\frac{\alpha}{A+1}+\frac{\beta}{B+1}\right)<\alpha a_{1}+\beta a_{2}+\frac{\alpha M}{A+1}+\frac{\beta N}{B+1} \leqslant 1+\frac{\alpha M}{A+1}+\frac{\beta N}{B+1},
$$

which implies that $m_{0}+a_{1}+a_{2}<2$ by Lemma 3.1

Let $\pi_{1}: S_{1} \rightarrow S$ be the blow up of the point $O$, and let $F_{1}$ be the $\pi_{1}$-exceptional curve. Then

$$
K_{S_{1}}+D^{1}+a_{1} \Delta_{1}^{1}+a_{2} \Delta_{2}^{1}+\left(m_{0}+a_{1}+a_{2}-1\right) F_{1} \sim_{\mathbb{Q}} \pi_{1}^{*}\left(K_{S}+D+a_{1} \Delta_{1}+a_{2} \Delta_{2}\right),
$$

where $D^{1}, \Delta_{1}^{1}, \Delta_{2}^{1}$ are proper transforms of the divisors $D, \Delta_{1}, \Delta_{2}$ via $\pi_{1}$, respectively. Then

$$
\left(S_{1}, D^{1}+a_{1} \Delta_{1}^{1}+a_{2} \Delta_{2}^{1}+\left(m_{0}+a_{1}+a_{2}-1\right) F_{1}\right)
$$

is not Kawamata log terminal at some point $O_{1} \in F_{1}$ (see Remark 2.4), where $m_{0}+a_{1}+a_{2} \geqslant 1$.

Lemma 3.5. Either $O_{1}=F_{1} \cap \Delta_{1}^{1}$ or $O_{1}=F_{1} \cap \Delta_{2}^{1}$. 
Proof. Suppose that $O_{1} \notin \Delta_{1}^{1} \cup \Delta_{2}^{1}$. Then $m_{0}=D^{1} \cdot F_{1}>1$ by Lemma 2.3. But

$$
m_{0}\left(\frac{\beta+B \alpha}{A B-1}+\frac{\alpha+A \beta}{A B-1}\right)<\left(M+A a_{1}-a_{2}\right) \frac{\beta+B \alpha}{A B-1}+\left(N+B a_{2}-a_{1}\right) \frac{\alpha+A \beta}{A B-1},
$$

because $m_{0}<M+A a_{1}-a_{2}$ and $m_{0}<N+B a_{2}-a_{1}$. On the other hand, we have

$$
\left(M+A a_{1}-a_{2}\right) \frac{\beta+B \alpha}{A B-1}+\left(N+B a_{2}-a_{1}\right) \frac{\alpha+A \beta}{A B-1} \leqslant 1+\frac{M \beta+M B \alpha+N \alpha+A N \beta}{A B-1},
$$

because $\alpha a_{1}+\beta a_{2} \leqslant 1$ and $A B-1>0$. But we already proved that $m_{0}>1$. Thus, we see that

$$
\beta+B \alpha+\alpha+A \beta<A B-1+M \beta+M B \alpha+N \alpha+A N \beta,
$$

which is impossible by Lemma 3.1 ,

Lemma 3.6. The inequality $O_{1} \neq F_{1} \cap \Delta_{1}^{1}$ holds.

Proof. Suppose that $O_{1}=F_{1} \cap \Delta_{1}^{1}$. It follows from Lemma 2.3 that

$$
M+A a_{1}-a_{2}-m_{0}>\operatorname{mult}_{O_{1}}\left(D^{1} \cdot \Delta_{1}^{1}\right)>1-\left(m_{0}+a_{1}+a_{2}-1\right),
$$

which implies that $a_{1}>(2-M) /(A+1)$. Then

$$
\frac{(2-M) \alpha}{A+1}+\frac{\beta(1-N)}{B}<\alpha a_{1}+\beta a_{2} \leqslant 1,
$$

because $a_{2}>(1-N) / B$ by Lemma 3.2, Thus, we see that

$$
\frac{(2-M) \alpha}{A+1}+\frac{\beta(1-N)}{B}<1
$$

which is impossible by Lemma 3.1 .

Therefore, we see that $O_{1}=F_{1} \cap \Delta_{2}^{1}$. Then the log pair

$$
\left(S_{1}, D^{1}+a_{1} \Delta_{1}^{1}+a_{2} \Delta_{2}^{1}+\left(m_{0}+a_{1}+a_{2}-1\right) F_{1}\right)
$$

is not Kawamata log terminal at the point $O_{1}$. We know that $1>m_{0}+a_{1}+a_{2}-1 \geqslant 0$.

We have a blow up $\pi_{1}: S_{1} \rightarrow S$. For any $n \in \mathbb{N}$, consider a sequence of blow ups

$$
S_{n} \longrightarrow \pi_{n} S_{n-1} \stackrel{\pi_{n-1}}{\longrightarrow} \cdots \stackrel{\pi_{3}}{\longrightarrow} S_{2} \stackrel{\pi_{2}}{\longrightarrow} S_{1} \stackrel{\pi_{1}}{\longrightarrow} S
$$

such that $\pi_{i+1}: S_{i+1} \rightarrow S_{i}$ is a blow up of the point $F_{i} \cap \Delta_{2}^{i}$ for every $i \in\{1, \ldots, n-1\}$, where

- we denote by $F_{i}$ the exceptional curve of the morphism $\pi_{i}$,

- we denote by $\Delta_{2}^{i}$ the proper transform of the curve $\Delta_{2}$ on the surface $S_{i}$.

For every $k \in\{1, \ldots, n\}$ and for every $i \in\{1, \ldots, k\}$, let $D^{k}, \Delta_{1}^{k}$ and $F_{i}^{k}$ be the proper transforms on the surface $S_{k}$ of the divisors $D, \Delta_{1}$ and $F_{i}$, respectively. Then

$$
K_{S_{n}}+D^{n}+a_{1} \Delta_{1}^{n}+a_{2} \Delta_{2}^{n}+\sum_{i=1}^{n}\left(a_{1}+i a_{2}-i+\sum_{j=0}^{i-1} m_{j}\right) F_{i}^{n} \sim_{\mathbb{Q}} \pi^{*}\left(K_{S}+D+a_{1} \Delta_{1}+a_{2} \Delta_{2}\right),
$$

where $\pi=\pi_{n} \circ \cdots \circ \pi_{2} \circ \pi_{1}$ and $m_{i}=$ mult $_{O_{i}}\left(D^{i}\right)$ for every $i \in\{1, \ldots, n\}$. Then the log pair

$$
\left(S_{n}, D^{n}+a_{1} \Delta_{1}^{n}+a_{2} \Delta_{2}^{n}+\sum_{i=1}^{n}\left(a_{1}+i a_{2}-i+\sum_{j=0}^{i-1} m_{j}\right) F_{i}^{n}\right)
$$

is not Kawamata log terminal at some point of the set $F_{1}^{n} \cup F_{2}^{n} \cup \cdots \cup F_{n}^{n}$ (see Remark 2.4).

Put $O_{k}=F_{k} \cap \Delta_{2}^{k}$ for every $k \in\{1, \ldots, n\}$. 
Lemma 3.8. For every $i \in\{1, \ldots, n\}$, we have

$$
1>a_{1}+i a_{2}-i+\sum_{j=0}^{i-1} m_{j} \geqslant 0
$$

and (3.7) is Kawamata log terminal at every point of the set $\left(F_{1}^{n} \cup F_{2}^{n} \cup \cdots \cup F_{n}^{n}\right) \backslash O_{n}$.

Since $\operatorname{mult}_{O}\left(D \cdot \Delta_{2}\right)<N+B a_{2}-a_{1}$ by assumption, it follows from Lemma 3.8 that

$$
N+B a_{2}-a_{1}>\operatorname{mult}_{O}\left(D \cdot \Delta_{2}\right) \geqslant \sum_{i=0}^{n-1} m_{i} \geqslant(n-1)\left(1-a_{2}\right)-a_{1},
$$

which implies that $n \leqslant\left(N+B a_{2}\right) /\left(1-a_{2}\right)$. On the other hand, the assertion of Lemma 3.8 holds for arbitrary $n \in \mathbb{N}$. So, taking any $n>\left(N+B a_{2}\right) /\left(1-a_{2}\right)$, we obtain a contradiction.

We see that to prove Theorem 1.28 , it is enough to prove Lemma 3.8 .

Let us prove Lemma 3.8 by induction on $n \in \mathbb{N}$. The case $n=1$ is already done.

We may assume that $n \geqslant 2$. For every $k \in\{1, \ldots, n-1\}$, we may assume that

$$
1>a_{1}+k a_{2}-k+\sum_{j=0}^{k-1} m_{j} \geqslant 0,
$$

the singularities of the log pair

$$
\left(S_{k}, D^{k}+a_{1} \Delta_{1}^{k}+a_{2} \Delta_{2}^{k}+\sum_{i=1}^{k}\left(a_{1}+k a_{2}-k+\sum_{j=0}^{i-1} m_{j}\right) F_{i}^{k}\right)
$$

are Kawamata log terminal along $\left(F_{1}^{k} \cup F_{2}^{k} \cup \cdots \cup F_{k}^{k}\right) \backslash O_{k}$ and not Kawamata log terminal at $O_{k}$.

Lemma 3.9. The inequality $a_{2}>(n-N) /(B+n-1)$ holds.

Proof. The singularities of the log pair

$$
\left(S_{n-1}, D^{n-1}+a_{2} \Delta_{2}^{n-1}+\left(a_{1}+(n-1) a_{2}-(n-1)+\sum_{j=0}^{n-2} m_{j}\right) F_{n-1}^{n-1}\right)
$$

are not Kawamata log terminal at the point $O_{n-1}$. Then it follows from Lemma 2.3 that

$$
N+B a_{2}-a_{1}-\sum_{j=0}^{n-2} m_{j}>\operatorname{mult}_{O_{n-1}}\left(D^{n-1} \cdot \Delta_{2}^{n-1}\right)>1-\left(a_{1}+(n-1) a_{2}-(n-1)+\sum_{j=0}^{n-2} m_{j}\right),
$$

which implies that $a_{2}>(n-N) /(B+n-1)$.

Lemma 3.10. The inequalities $1>a_{1}+n a_{2}-n+\sum_{j=0}^{n-1} m_{j} \geqslant 0$ hold.

Proof. The inequality $a_{1}+n a_{2}-n+\sum_{j=0}^{n-1} m_{j} \geqslant 0$ follows from the fact that the log pair

$$
\left(S_{n-1}, D^{n-1}+a_{2} \Delta_{2}^{n-1}+\left(a_{1}+(n-1) a_{2}-(n-1)+\sum_{j=0}^{n-2} m_{j}\right) F_{n-1}^{n-1}\right)
$$

is not Kawamata log terminal at the point $O_{n-1}$.

Suppose that $a_{1}+n a_{2}-n+\sum_{j=0}^{n-1} m_{j} \geqslant 1$. Let us derive a contradiction.

It follows from Remark 3.3 that $m_{0}+a_{2} \leqslant M+A a_{1}$. Then

$$
a_{1}+n M+n A a_{1}-n \geqslant a_{1}+n a_{2}-n+n m_{0} \geqslant a_{1}+n a_{2}-n+\sum_{j=0}^{n-1} m_{j} \geqslant 1,
$$


which implies that $a_{1} \geqslant(n+1-M n) /(n A+1)$. But $a_{2}>(n-N) /(B+n-1)$ by Lemma 3.9. Then

$$
\left(\frac{\alpha(1-M)}{A}+\beta\right)+\alpha \frac{A-1+M}{A(A n+1)}+\beta \frac{1-B-N}{B+n-1}=\alpha \frac{n+1-M n}{n A+1}+\beta \frac{n-N}{B+n-1}<\alpha a_{1}+\beta a_{2} \leqslant 1,
$$

where $\alpha(1-M) / A+\beta \geqslant 1$ by assumption. Therefore, we see that

$$
\alpha \frac{A+M-1}{A(A n+1)}<\beta \frac{B+N-1}{B+n-1},
$$

where $n \geqslant 2$. But $A+M>1$ and $B+N>1$ by Lemma 3.2. since $a_{1}<1$ and $a_{2}<1$. Then

$$
\frac{A(A n+1)}{\alpha(A+M-1)}>\frac{B+n-1}{\beta(B+N-1)},
$$

but $A^{2}(B+N-1) \beta \leqslant \alpha(A+M-1)$ by assumption. Then

$\frac{A}{\alpha(A+M-1)}-\frac{B-1}{\beta(B+N-1)} \geqslant\left(\frac{A^{2}}{\alpha(A+M-1)}-\frac{1}{\beta(B+N-1)}\right) n+\frac{A}{\alpha(A+M-1)}-\frac{B-1}{\beta(B+N-1)}>0$,

which implies that $\beta A(B+N-1)>\alpha(B-1)(A+M-1)$. Then

$$
\frac{\alpha(A+M-1)}{A} \geqslant \beta A(B+N-1)>\alpha(B-1)(A+M-1)
$$

because $A^{2}(B+N-1) \beta \leqslant \alpha(A+M-1)$ by assumption. Then we have $\alpha \neq 0$ and $A(B-1)<1$, which is impossible, because $A(B-1) \geqslant 1$ by assumption.

Lemma 3.11. The log pair (3.7) is Kawamata log terminal at every point of the set

$$
F_{n} \backslash\left(\left(F_{n} \cap F_{n-1}^{n}\right) \bigcup\left(F_{n} \cap \Delta_{2}^{n}\right)\right) \text {. }
$$

Proof. Suppose that there is a point $Q \in F_{n}$ such that

$$
F_{n} \cap F_{n-1}^{n} \neq Q \neq F_{n} \cap \Delta_{2}^{n},
$$

but (3.7) is not Kawamata $\log$ terminal at the point $Q$. Then the log pair

$$
\left(S_{n}, D^{n}+\left(a_{1}+n a_{2}-n+\sum_{j=0}^{n-1} m_{j}\right) F_{n}\right)
$$

is not Kawamata $\log$ terminal at the point $Q$ as well. Then

$$
m_{0} \geqslant m_{n-1}=D^{n} \cdot F_{n}>1
$$

by Lemma 2.3 , because $a_{1}+n a_{2}-n+\sum_{j=0}^{n-1} m_{j}<1$ by Lemma 3.10 , Then

$$
m_{0}\left(\frac{\beta+B \alpha}{A B-1}+\frac{\alpha+A \beta}{A B-1}\right)<\left(M+A a_{1}-a_{2}\right) \frac{\beta+B \alpha}{A B-1}+\left(N+B a_{2}-a_{1}\right) \frac{\alpha+A \beta}{A B-1},
$$

because $m_{0}<M+A a_{1}-a_{2}$ and $m_{0}<N+B a_{2}-a_{1}$ by Remark 3.3. We have

$$
\left(M+A a_{1}-a_{2}\right) \frac{\beta+B \alpha}{A B-1}+\left(N+B a_{2}-a_{1}\right) \frac{\alpha+A \beta}{A B-1} \leqslant 1+\frac{M \beta+M B \alpha+N \alpha+A N \beta}{A B-1},
$$

because $\alpha a_{1}+\beta a_{2} \leqslant 1$ and $A B-1>0$. But $m_{0}>1$. Thus, we see that

$$
\beta+B \alpha+\alpha+A \beta<A B-1+M \beta+M B \alpha+N \alpha+A N \beta,
$$

which contradicts our initial assumptions.

Lemma 3.12. The log pair (3.7) is Kawamata log terminal at the point $F_{n} \cap F_{n-1}^{n}$. 
Proof. Suppose that (3.7) is not Kawamata log terminal at $F_{n} \cap F_{n-1}^{n}$. Then the log pair

$$
\left(S_{n}, D^{n}+\left(a_{1}+(n-1) a_{2}-(n-1)+\sum_{j=0}^{n-2} m_{j}\right) F_{n-1}^{n}+\left(a_{1}+n a_{2}-n+\sum_{j=0}^{n-1} m_{j}\right) F_{n}\right)
$$

is not Kawamata log terminal at the point $F_{n} \cap F_{n-1}^{n}$ as well. Then

$$
m_{n-2}-m_{n-1}=D^{n} \cdot F_{n-2}>1-\left(a_{1}+n a_{2}-n+\sum_{j=0}^{n-1} m_{j}\right)
$$

by Lemma 2.3 , because $a_{1}+(n-1) a_{2}-(n-1)+\sum_{j=0}^{n-2} m_{j}<1$. Note that

$$
M+A a_{1}-a_{2}-m_{0}>\operatorname{mult}_{O}\left(D \cdot \Delta_{1}\right)-m_{0} \geqslant \operatorname{mult}_{O}(D) \operatorname{mult}_{O}\left(\Delta_{1}\right)-m_{0}=0,
$$

which implies that $m_{0}+a_{2}<A a_{1}+M$. Then

$$
n M+n A a_{1}-n a_{2}>n m_{0} \geqslant m_{n-2}-m_{n-1}+\sum_{j=0}^{n-1} m_{j}>n+1-a_{1}-n a_{2},
$$

which gives $a_{1}>(n+1-n M) /(A n+1)$.

Now arguing as in the proof of Lemma 3.10, we obtain a contradiction.

The assertion of Lemma 3.8 is proved. The assertion of Theorem 1.28 is proved.

\section{ONE CYCLIC SINGULAR POINT}

Let $X$ be a sextic surface in $\mathbb{P}(1,1,2,3)$ with canonical singularities such that $|\operatorname{Sing}(X)|=1$, let $\omega: X \rightarrow \mathbb{P}(1,1,2)$ be the natural double cover, let $R$ be its ramification curve in $\mathbb{P}(1,1,2)$, and suppose that $\operatorname{Sing}(X)$ consists of one singular point of type $\mathbb{A}_{m}$, where $m \in\{1, \ldots, 8\}$.

Theorem 4.1. The following equality holds:

$$
\operatorname{lct}(X)=\left\{\begin{array}{l}
\operatorname{lct}_{3}(X)=1 / 2 \text { if } m=8, \\
\operatorname{lct}_{2}(X)=1 / 2 \text { if } m=7 \text { and } R \text { is reducible, } \\
\operatorname{lct}_{3}(X)=3 / 5 \text { if } m=7 \text { and } R \text { is irreducible, } \\
\operatorname{lct}_{2}(X)=2 / 3 \text { if } m=6, \\
\operatorname{lct}_{2}(X)=2 / 3 \text { if } m=5, \\
\operatorname{lct}_{2}(X)=4 / 5 \text { if } m=4, \\
\operatorname{lct}_{1}(X) \text { in the remaining cases, }
\end{array}\right.
$$

and if $\operatorname{lct}(X)=2 / 3$, then there is a unique effective $\mathbb{Q}$-divisor $D$ on $X$ such that $D \sim_{\mathbb{Q}}-K_{X}$ and

$$
\mathrm{c}(X, D)=\operatorname{lct}(X)=\frac{2}{3}
$$

By Theorem 1.5. Corollary 1.12 and Remark 1.8, we obtain the following two corollaries.

Corollary 4.2. If $m \leqslant 6$, then $\operatorname{lct}_{n, 2}(X)>2 / 3$ for every $n \in \mathbb{N}$.

Corollary 4.3. If $m \leqslant 6$, then $X$ is Kähler-Enstein.

In the rest of this section we will prove Theorem 4.1 .

Let $D$ be an arbitrary effective $\mathbb{Q}$-divisor on the surface $X$ such that

$$
D \sim_{\mathbb{Q}}-K_{X}
$$


and put $\mu=\mathrm{c}(X, D)$. To prove Theorem 4.1, it is enough to show that

$$
\mu \geqslant\left\{\begin{array}{l}
\operatorname{lct}_{3}(X)=1 / 2 \text { if } m=8, \\
\operatorname{lct}_{2}(X)=1 / 2 \text { if } m=7 \text { and } R \text { is reducible, } \\
\operatorname{lct}_{3}(X)=3 / 5 \text { if } m=7 \text { and } R \text { is irreducible, } \\
\operatorname{lct}_{2}(X)=2 / 3 \text { if } m=6, \\
\operatorname{lct}_{2}(X)=2 / 3 \text { if } m=5, \\
\operatorname{lct}_{2}(X)=4 / 5 \text { if } m=4, \\
\operatorname{lct}_{1}(X) \text { in the remaining cases, }
\end{array}\right.
$$

and if $\mu=\operatorname{lct}(X)=2 / 3$, then $D$ is uniquely defined. Note that $\operatorname{lct}_{1}(X) \geqslant 5 / 6$ if $m \geqslant 3$ (see [30]).

Let us prove Theorem 4.1. By Lemma 2.6, we may assume that $m \geqslant 3$ and $\mu<\operatorname{lct}_{1}(X)$. Then

$$
\operatorname{LCS}(X, \mu D)=\operatorname{Sing}(X)
$$

by Lemma 2.6. Put $P=\operatorname{Sing}(X)$.

Let $\pi: \bar{X} \rightarrow X$ be a minimal resolution, let $E_{1}, E_{2}, \ldots, E_{m}$ be $\pi$-exceptional curves such that

$$
E_{i} \cdot E_{j} \neq 0 \Longleftrightarrow|i-j| \leqslant 1
$$

let $C$ be the curve in $\left|-K_{X}\right|$ such that $P \in C$, and let $\bar{C}$ be it proper transform on $\bar{X}$. Then

$$
\bar{C} \sim_{\mathbb{Q}} \pi^{*}(C)-\sum_{i=1}^{m} E_{i},
$$

and the curve $C$ is irreducible. We may assume that $D \neq C$, because $\mu \geqslant \operatorname{lct}_{1}(X)$ if $D=C$.

By Remark 2.1, we may assume that $C \not \subset \operatorname{Supp}(D)$.

Let $\bar{D}$ be the proper transform of the divisor $D$ on the surface $\bar{X}$. Then

$$
\bar{D} \sim_{\mathbb{Q}} \pi^{*}(D)-\sum_{i=1}^{m} a_{i} E_{i},
$$

where $a_{i}$ is a non-negative rational number. Then the log pair

$$
\left(\bar{X}, \mu \bar{D}+\sum_{i=1}^{m} \mu a_{i} E_{i}\right)
$$

is not Kawamata log terminal (by Remark 2.4). On the other hand, we have

$$
\bar{D} \cdot E_{1}=2 a_{1}-a_{2}, \bar{D} \cdot E_{2}=2 a_{2}-a_{1}-a_{3}, \cdots, \bar{D} \cdot E_{m-1}=2 a_{m-1}-a_{m-2}-a_{m}, \bar{D} \cdot E_{m}=2 a_{m}-a_{m-1},
$$

where all intersections $\bar{D} \cdot E_{1}, \bar{D} \cdot E_{2}, \ldots, \bar{D} \cdot E_{m}$ are non-negative. Moreover, we have

$$
\bar{D} \cdot \bar{C}=1-a_{1}-a_{m}
$$


where the intersection $\bar{D} \cdot \bar{C}$ is non-negative, since $C \not \subset \operatorname{Supp}(D)$ by assumption. Hence, we have

$$
\left\{\begin{array}{l}
a_{1} \geqslant \frac{a_{2}}{2} \\
a_{2} \geqslant \frac{a_{1}+a_{3}}{2} \\
a_{3} \geqslant \frac{a_{2}+a_{4}}{2} \\
\cdots \\
a_{m-1} \geqslant \frac{a_{m-2}+a_{m}}{2} \\
a_{m} \geqslant \frac{a_{m-1}}{2} \\
1 \geqslant a_{1}+a_{m}
\end{array}\right.
$$

It should be pointed out that at least one inequality in (4.5) must be strict, since $\bar{D} \cdot E_{i}>0$ for at least one $i \in\{1, \ldots, m\}$, because $P \in \operatorname{Supp}(D)$. Then $a_{i}>0$ for some $i \in\{1, \ldots, m\}$.

Note that $a_{1} \geqslant a_{2} / 2$ by (4.5). Similarly, it follows from (4.5) that

$$
a_{2} \geqslant \frac{a_{1}+a_{3}}{2} \geqslant \frac{a_{1}}{4}+\frac{a_{3}}{4}
$$

which implies that $a_{2} \geqslant 2 a_{3} / 3$. Arguing in the same way, we see that

$$
a_{k} \geqslant \frac{k}{k+1} a_{k+1}
$$

for every $k \in\{1, \ldots, m-1\}$ (use (4.5) and induction on $k$ ). Using symmetry, we see that

$$
a_{k+1} \geqslant \frac{m-k}{m-k+1} a_{k}
$$

for every $k \in\{1, \ldots, m-1\}$. In particular, the inequality $a_{k}>0$ holds for every $k \in\{1, \ldots, m\}$, since we already know that $a_{i}>0$ for some $i \in\{1, \ldots, m\}$.

Lemma 4.6. Suppose that $\mu a_{i}<1$ for every $i \in\{1, \ldots, m\}$. Then

- there exists a point

$$
Q \in\left\{E_{1} \cap E_{2}, E_{2} \cap E_{3}, \ldots, E_{m-1} \cap E_{m}\right\}
$$

such that the log pair (4.4) is not Kawamata log terminal at $Q$,

- the log pair (4.4) is Kawamata log terminal outside of the point $Q$,

- if $\mu<(m+1) /(2 m-2)$, then $Q \neq E_{1} \cap E_{2}$ and $Q \neq E_{m-1} \cap E_{m}$.

Proof. It follows from Remark 2.4 and Theorem 2.2 that there is a point $Q \in \cup_{i=1}^{m} E_{i}$ such that the log pair (4.4) is not Kawamata log terminal at $Q$ and is Kawamata log terminal elsewhere.

Suppose that $Q \in E_{1}$ and $Q \notin E_{2}$. Then

$$
2 a_{1}-a_{2}=\bar{D} \cdot E_{i}>1
$$


by Lemma 2.3. Taking (4.5) into account, we get

$$
\left\{\begin{array}{l}
a_{1}>\frac{1}{2}+\frac{a_{2}}{2}, \\
a_{2} \geqslant \frac{a_{1}+a_{3}}{2}, \\
a_{3} \geqslant \frac{a_{2}+a_{4}}{2}, \\
\cdots \\
a_{m-1} \geqslant \frac{a_{m-2}+a_{m}}{2}, \\
a_{m} \geqslant \frac{a_{m-1}}{2}
\end{array}\right.
$$

and adding all these inequalities together we get

$$
\sum_{i=1}^{m} a_{i}>\frac{1}{2}+\frac{a_{1}}{2}+\sum_{i=2}^{m-1} a_{i}+\frac{a_{m}}{2},
$$

which implies that $a_{1}+a_{m}>1$. However, the later is impossible, since $a_{1}+a_{m} \leqslant 1$ by (4.5).

We see that if $Q \in E_{1}$, then $Q=E_{1} \cap E_{2}$. Similarly, we see that $Q=E_{m-1} \cap E_{m}$ if $Q \in E_{m}$.

Suppose that $Q \in E_{i}$ and $Q \notin E_{j}$ for every $j \neq i$. Then $i \neq 1$ and $i \neq m$. We have

$$
2 a_{i}-a_{i-1}-a_{i+1}=\bar{D} \cdot E_{i}>1
$$

by Lemma 2.3. Taking (4.5) into account, we get

$$
\left\{\begin{array}{l}
a_{1}>\frac{a_{2}}{2} \\
a_{2} \geqslant \frac{a_{1}+a_{3}}{2}, \\
a_{3} \geqslant \frac{a_{2}+a_{4}}{2} \\
\ldots \\
a_{i} \geqslant \frac{1}{2}+\frac{a_{i-1}+a_{i+1}}{2}, \\
\ldots \\
a_{m-1} \geqslant \frac{a_{m-2}+a_{m}}{2} \\
a_{m} \geqslant \frac{a_{m-1}}{2}
\end{array}\right.
$$

and adding all these inequalities together we get

$$
\sum_{i=1}^{m} a_{i}>\frac{1}{2}+\frac{a_{1}}{2}+\sum_{i=2}^{m-1} a_{i}+\frac{a_{m}}{2},
$$

which implies that $a_{1}+a_{m}>1$. However, the later is impossible, since $a_{1}+a_{m} \leqslant 1$ by (4.5).

Thus, we see that there is $k \in\{1, \ldots, m-1\}$ such that $Q=E_{k} \cap E_{k+1}$.

Suppose that $\mu<(m+1) /(2 m-2)$. Let us show that $k \neq 1$ and $k \neq m-1$.

Due to symmetry, it is enough to show that $k \neq 1$. Recall that $m \geqslant 3$.

Suppose that $k=1$. Then $Q=E_{1} \cap E_{2}$. Take $\bar{\mu} \in \mathbb{Q}$ such that $(m+1) /(2 m-2)>\bar{\mu}>\mu$ and

$$
\left(\bar{X}, \mu \bar{D}+\bar{\mu} a_{1} E_{1}+\bar{\mu} a_{2} E_{2}\right)
$$


is not Kawamata log terminal at $Q$ and is Kawamata log terminal outside of the point $Q$. Then

$$
\frac{2 m-2}{m+1} \bar{\mu} a_{1}+\frac{2}{m+1} \bar{\mu} a_{2}<a_{1}+\frac{1}{m-1} a_{2} \leqslant 1,
$$

by (4.5), since $a_{1} \neq 0$ and $a_{2} \neq 0$. On the other hand, we have

$$
\operatorname{mult}_{Q}\left(\mu \bar{D} \cdot E_{1}\right) \leqslant \mu \bar{D} \cdot E_{1}=\mu\left(2 a_{1}-a_{2}\right)<\bar{\mu}\left(2 a_{1}-a_{2}\right),
$$

since $\mu<\bar{\mu}$. Therefore, it follows from Corollary 1.29 that

$$
\mu\left(2 a_{2}-a_{1}-a_{3}\right)=\mu \bar{D} \cdot E_{2} \geqslant \operatorname{mult}_{Q}\left(\mu \bar{D} \cdot E_{2}\right) \geqslant \frac{m}{m-1} \bar{\mu} a_{2}-\bar{\mu} a_{1},
$$

which implies that $a_{2}(m-2)>a_{3}(m-1)$, since $\mu<\bar{\mu}$. But we proved earlier that

$$
a_{3} \geqslant \frac{m-2}{m-1} a_{2}
$$

which is impossible, since $a_{2}(m-2)>a_{3}(m-1)$. Thus, we see that $k \neq 1$.

If $m=3$, then it follows from (4.5) that $a_{1} \leqslant 3 / 4, a_{2} \leqslant 1, a_{3} \leqslant 3 / 4$.

Corollary 4.7. If $m=3$, then $\mu \geqslant \operatorname{lct}_{1}(X) \geqslant 5 / 6$.

Lemma 4.8. Suppose that $m=4$. Then $\mu \geqslant \operatorname{lct}_{2}(X)=4 / 5$.

Proof. There is a unique smooth irreducible curve $\bar{Z} \subset \bar{X}$ such that

$$
\bar{Z} \sim \pi^{*}\left(-2 K_{X}\right)-E_{1}-2 E_{2}-2 E_{3}-E_{4}
$$

and $E_{2} \cap E_{3} \in \bar{Z}$ (cf. the proof of Lemma $\underline{6.9}$ ). Put $Z=\pi(\bar{Z})$. Then

$$
\operatorname{lct}_{2}(X) \leqslant \mathrm{c}\left(X, \frac{1}{2} Z\right)=\frac{4}{5} .
$$

To complete the proof, it is enough to show that $\mu \geqslant 4 / 5$. Suppose that $\mu<4 / 5$.

By Remark 2.1, we may assume that $Z \not \subset \operatorname{Supp}(D)$, because $Z$ is irreducible.

It follows from (4.5) that $a_{1} \leqslant 4 / 5, a_{2} \leqslant 6 / 5, a_{3} \leqslant 6 / 5, a_{4} \leqslant 4 / 5$.

Put $Q=E_{2} \cap E_{3}$. Then it follows from Lemma 4.6 that (4.4) is not Kawamata log terminal at the point $Q$ and is Kawamata $\log$ terminal outside of the point $Q$. Then

$$
2 a_{2}-\frac{1}{2} a_{2}-a_{3} \geqslant 2 a_{2}-a_{1}-a_{3}=\bar{D} \cdot E_{2} \geqslant \operatorname{mult}_{Q}\left(\bar{D} \cdot E_{2}\right)>\frac{5}{4}-a_{3},
$$

by Lemma 2.3. Similarly, we see that

$$
2 a_{3}-a_{2}-a_{4}=\bar{D} \cdot E_{3} \geqslant \operatorname{mult}_{Q}\left(\bar{D} \cdot E_{3}\right)>\frac{5}{4}-a_{2},
$$

which implies that $a_{2}>5 / 6$ and $a_{3}>5 / 6$.

Let $\xi: \tilde{X} \rightarrow \bar{X}$ be a blow up of the point $Q$, let $E$ be the exceptional curve of the blow up $\xi$, and let $\tilde{D}$ be the proper transform of the divisor $\bar{D}$ on the surface $\tilde{X}$. Put $\delta=\operatorname{mult}_{Q}(\bar{D})$.

Let $\tilde{E}_{1}, \tilde{E}_{2}, \tilde{E}_{3}, \tilde{E}_{4}$ be the proper transforms on $\tilde{X}$ of $E_{1}, E_{2}, E_{3}, E_{4}$, respectively. Then

$$
\left(\tilde{X}, \mu \tilde{D}+\mu a_{2} \tilde{E}_{2}+\mu a_{3} \tilde{E}_{3}+\left(\mu a_{2}+\mu a_{3}+\mu \delta-1\right) E\right)
$$

is not Kawamata log terminal at some point $O \in E$.

Let $\tilde{Z}$ be the proper transform on $\tilde{X}$ of the curve $\bar{Z}$. Then

$$
0 \leqslant \tilde{Z} \cdot \tilde{D}=2-a_{2}-a_{3}-\operatorname{mult}_{Q}(\bar{D})=2-a_{2}-a_{3}-\delta
$$


which implies that $\delta+a_{2}+a_{3} \leqslant 2$. We have $\mu a_{2}+\mu a_{3}+\mu \delta-1 \leqslant 2 \mu-1 \leqslant 3 / 5$, which implies that (4.9) is Kawamata log terminal outside of the point $O$ by Theorem 2.2. We have

$$
\left\{\begin{array}{l}
2 a_{3}-a_{2}-a_{4}-\delta=\tilde{E}_{3} \cdot \tilde{D} \geqslant 0, \\
2 a_{2}-a_{1}-a_{3}-\delta=\tilde{E}_{2} \cdot \tilde{D} \geqslant 0,
\end{array}\right.
$$

which implies that $\delta \leqslant 1$. If $O \notin \tilde{E}_{2} \cup \tilde{E}_{3}$, then

$$
1 \geqslant \delta=\tilde{D} \cdot E \geqslant \operatorname{mult}_{O}(\tilde{D} \cdot E)>\frac{5}{4}
$$

by Lemma 2.3. Thus, we see that either $O=\tilde{E}_{2} \cap E$ or $O=\tilde{E}_{3} \cap E$.

Without loss of generality, we may assume that $O=\tilde{E}_{2} \cap E$. By Lemma 2.3, one has

$$
\frac{5}{4}-a_{2}>\frac{7}{6}-a_{2}=2-\frac{5}{6}-a_{2}>2-a_{2}-a_{3} \geqslant \delta=\tilde{D} \cdot E \geqslant \operatorname{mult}_{O}(\tilde{D} \cdot E)>\frac{5}{4}-a_{2},
$$

since $\delta+a_{2}+a_{3} \leqslant 2$ and $a_{3}>5 / 6$. The obtained contradiction concludes the proof.

Let $\tau$ be a biregular involution of the surface $\bar{X}$ that is induced by the double cover $\omega$.

Lemma 4.10. Suppose that $m=5$. Then there exists a unique curve $Z \in\left|-2 K_{X}\right|$ such that

$$
\mathrm{c}\left(X, \frac{1}{2} Z\right)=\operatorname{lct}_{2}(X)=\frac{2}{3},
$$

and either $D=Z / 2$ or $\mu>2 / 3$.

Proof. Let $\alpha: \bar{X} \rightarrow \breve{X}$ be a contraction of the curves $\bar{C}, E_{5}, E_{4}, E_{3}$. Then

$$
\alpha\left(E_{1}\right) \cdot \alpha\left(E_{1}\right)=\alpha\left(E_{2}\right) \cdot \alpha\left(E_{2}\right)=-1,
$$

and $\breve{X}$ is a smooth del Pezzo surface such that $K_{\breve{X}}^{2}=5$, which implies that there is a smooth irreducible rational curve $\breve{L}_{2}$ on the surface $\breve{X}$ such that $\breve{L}_{2} \cdot \alpha\left(E_{2}\right)=1$ and $\breve{L}_{2} \cdot \breve{L}_{2}=-1$.

Let $\bar{L}_{2}$ be the proper transform of the curve $\breve{L}_{2}$ on the surface $\bar{X}$. Then $\bar{L}_{2} \cdot \bar{L}_{2}=-1$ and

$$
-K_{\bar{X}} \cdot \bar{L}_{2}=E_{2} \cdot \bar{L}_{2}=1 \text {, }
$$

which implies that $E_{1} \cdot \bar{L}_{2}=E_{3} \cdot \bar{L}_{2}=E_{4} \cdot \bar{L}_{2}=E_{5} \cdot \bar{L}_{2}=\bar{C} \cdot \bar{L}_{2}=0$.

Let $\beta: \bar{X} \rightarrow \check{X}$ be a contraction of the curves $\bar{L}_{2}, \bar{C}, E_{5}, E_{4}$. Then

$$
\beta\left(E_{2}\right) \cdot \beta\left(E_{2}\right)=\beta\left(E_{3}\right) \cdot \beta\left(E_{3}\right)=-1,
$$

and $\check{X}$ is a smooth del Pezzo surface such that $K_{\check{X}}^{2}=5$, which implies that there is an irreducible smooth curve $\check{L}_{3} \subset \check{X}$ such that $\check{L}_{3} \cdot \beta\left(E_{3}\right)=1$ and $\check{L}_{3} \cdot \check{L}_{3}=-1$ (cf. the proof of Lemma 6.8).

Let $\bar{L}_{3}$ be the proper transform of the curve $\check{L}_{3}$ on the surface $\bar{X}$. Then $\bar{L}_{3} \cdot \bar{L}_{3}=-1$ and

$$
-K_{\bar{X}} \cdot \bar{L}_{3}=E_{3} \cdot \bar{L}_{3}=1,
$$

which implies that $E_{1} \cdot \bar{L}_{3}=E_{2} \cdot \bar{L}_{3}=E_{4} \cdot \bar{L}_{3}=E_{5} \cdot \bar{L}_{3}=\bar{C} \cdot \bar{L}_{3}=0$.

If $\tau\left(\bar{L}_{3}\right)=\bar{L}_{3}$, then $2 \pi\left(\bar{L}_{3}\right) \sim-2 K_{X}$, but $\pi\left(\bar{L}_{3}\right)$ is not a Cartier divisor.

Put $Z=\pi\left(\bar{L}_{3}+\tau\left(\bar{L}_{3}\right)\right)$. Then $Z \sim-2 K_{X}$ and $\mathrm{c}(X, Z)=1 / 3$. We see that $\operatorname{lct}_{2}(X) \leqslant 2 / 3$.

Suppose that $D \neq Z / 2$. To complete the proof, it is enough to show that $\mu>2 / 3$.

Suppose that $\mu \leqslant 2 / 3$. Let us derive a contradiction. It follows from (4.5) that

$$
a_{1} \leqslant \frac{5}{6}, a_{2} \leqslant \frac{4}{3}, a_{3} \leqslant \frac{3}{2}, a_{4} \leqslant \frac{4}{3}, a_{5} \leqslant \frac{5}{6} .
$$

By Remark 2.1, without loss of generality we may assume that $\pi\left(\bar{L}_{3}\right) \not \subset \operatorname{Supp}(D)$. Then

$$
1-a_{3}=\bar{L}_{3} \cdot \bar{D} \geqslant 0,
$$

which implies that $a_{3} \leqslant 1$. 
Put $Q=E_{2} \cap E_{3}$. By Lemma 4.6, we may assume that (4.4) is not Kawamata log terminal at the point $Q$ and is Kawamata $\log$ terminal outside of the point $Q$. Then

$$
2 a_{3}-a_{2}-a_{4}=\bar{D} \cdot E_{3} \geqslant \operatorname{mult}_{Q}\left(\bar{D} \cdot E_{3}\right) \geqslant \frac{1}{\mu}-a_{2}>\frac{3}{2}-a_{2}
$$

by Lemma 2.3, which implies that $a_{3}>9 / 8$ by (4.5). But $a_{3} \leqslant 1$.

Lemma 4.11. Suppose that $m=6$. Then there exists a unique curve $Z \in\left|-2 K_{X}\right|$ such that

$$
\mathrm{c}\left(X, \frac{1}{2} Z\right)=\operatorname{lct}_{2}(X)=\frac{2}{3}
$$

and either $D=Z / 2$ or $\mu>2 / 3$.

Proof. Let $\alpha: \bar{X} \rightarrow \breve{X}$ be a contraction of the curves $\bar{C}, E_{6}, E_{5}, E_{4}$ and $E_{3}$. Then

$$
\alpha\left(E_{1}\right) \cdot \alpha\left(E_{1}\right)=\alpha\left(E_{2}\right) \cdot \alpha\left(E_{2}\right)=-1,
$$

and $\breve{X}$ is a smooth del Pezzo surface such that $K_{\breve{X}}^{2}=6$, which implies that there is a smooth irreducible rational curve $\breve{L}_{2}$ on the surface $\breve{X}$ such that $\breve{L}_{2} \cdot \alpha\left(E_{2}\right)=1$ and $\breve{L}_{2} \cdot \breve{L}_{2}=-1$.

Let $\bar{L}_{2}$ be the proper transform of the curve $\breve{L}_{2}$ on the surface $\bar{X}$. Then $\bar{L}_{2} \cdot \bar{L}_{2}=-1$ and

$$
-K_{\bar{X}} \cdot \bar{L}_{2}=E_{2} \cdot \bar{L}_{2}=1 \text {, }
$$

which implies that $E_{1} \cdot \bar{L}_{2}=E_{3} \cdot \bar{L}_{2}=E_{4} \cdot \bar{L}_{2}=E_{5} \cdot \bar{L}_{2}=E_{6} \cdot \bar{L}_{2}=\bar{C} \cdot \bar{L}_{2}=0$.

Let $\beta: \bar{X} \rightarrow \check{X}$ be a contraction of the curves $\bar{L}_{2}, \bar{C}, E_{6}, E_{5}$ and $E_{4}$. Then

$$
\beta\left(E_{2}\right) \cdot \beta\left(E_{2}\right)=\beta\left(E_{3}\right) \cdot \beta\left(E_{3}\right)=-1,
$$

and $\check{X}$ is a smooth del Pezzo surface such that $K_{\check{X}}^{2}=6$, which implies that there are irreducible smooth rational curves $\check{L}_{3}$ and $\check{L}_{2}^{\prime}$ on the surface $\check{X}$ such that

$$
\check{L}_{3} \cdot \beta\left(E_{3}\right)=\check{L}_{2}^{\prime} \cdot \beta\left(E_{2}\right)=1
$$

and $\check{L}_{3} \cdot \check{L}_{3}=\check{L}_{2}^{\prime} \cdot \check{L}_{2}^{\prime}=-1$. Let $\bar{L}_{3}$ and $\bar{L}_{2}^{\prime}$ be the proper transforms of the curves $\check{L}_{3}$ and $\check{L}_{2}^{\prime}$ on the surface $\bar{X}$, respectively. Then $\bar{L}_{3} \cdot \bar{L}_{3}=\bar{L}_{2}^{\prime} \cdot \bar{L}_{2}^{\prime}=-1$ and

$$
-K_{\bar{X}} \cdot \bar{L}_{3}=-K_{\bar{X}} \cdot \bar{L}_{2}^{\prime}=E_{3} \cdot \bar{L}_{3}=E_{2} \cdot \bar{L}_{2}^{\prime}=1,
$$

which implies that $\bar{C} \cdot \bar{L}_{3}=\bar{C} \cdot \bar{L}_{2}^{\prime}=0$, and $E_{i} \cdot \bar{L}_{3}=E_{j} \cdot \bar{L}_{2}^{\prime}=0$ for every $i \neq 3$ and $j \neq 2$,

Put $\bar{L}_{4}=\tau\left(\bar{L}_{3}\right), \bar{L}_{5}=\tau\left(\bar{L}_{2}\right), \bar{L}_{5}^{\prime}=\tau\left(\bar{L}_{2}^{\prime}\right)$. Then $\bar{C} \cdot \bar{L}_{4}=\bar{C} \cdot \bar{L}_{5}=\bar{C} \cdot \bar{L}_{5}^{\prime}=0$ and

$$
-K_{\bar{X}} \cdot \bar{L}_{4}=-K_{\bar{X}} \cdot \bar{L}_{5}=-K_{\bar{X}} \cdot \bar{L}_{5}^{\prime}=E_{4} \cdot \bar{L}_{4}=E_{5} \cdot \bar{L}_{5}=E_{5} \cdot \bar{L}_{5}^{\prime}=1 \text {, }
$$

which implies that $E_{i} \cdot \bar{L}_{5}=E_{i} \cdot \bar{L}_{5}^{\prime}=E_{j} \cdot \bar{L}_{4}=0$ for every $i \neq 5$ and $j \neq 4$.

Put $L_{3}=\pi\left(\bar{L}_{3}\right), L_{4}=\pi\left(\bar{L}_{4}\right), L_{2}=\pi\left(\bar{L}_{2}\right), L_{2}^{\prime}=\pi\left(\bar{L}_{2}^{\prime}\right), L_{5}=\pi\left(\bar{L}_{5}\right), L_{5}^{\prime}=\pi\left(\bar{L}_{5}^{\prime}\right)$. Then

$$
L_{3}+L_{4} \sim L_{2}+L_{5} \sim L_{2}^{\prime}+L_{5}^{\prime} \sim-2 K_{X},
$$

and $\mathrm{c}\left(X, L_{3}+L_{4}\right)=1 / 3$, which implies that $\operatorname{lct}_{2}(X) \leqslant 2 / 3$.

Note that $\mathrm{c}\left(X, L_{2}+L_{5}\right)=\mathrm{c}\left(X, L_{2}^{\prime}+L_{5}^{\prime}\right)=1 / 2$.

Suppose that $D \neq\left(L_{3}+L_{4}\right) / 2$. To complete the proof, it is enough to show that $\mu>2 / 3$.

Suppose that $\mu \leqslant 2 / 3$. Let us derive a contradiction.

It follows from (4.5) that $a_{1} \leqslant 6 / 7, a_{2} \leqslant 10 / 7, a_{3} \leqslant 12 / 7, a_{4} \leqslant 12 / 7, a_{5} \leqslant 10 / 7, a_{6} \leqslant 6 / 7$.

By Remark 2.1, without loss of generality we may assume that $\bar{L}_{4} \not \subset \operatorname{Supp}(D)$. Then

$$
1-a_{4}=\bar{L}_{3} \cdot \bar{D} \geqslant 0,
$$

which gives us $a_{4} \leqslant 1$. Similarly, we may assume that either $\bar{L}_{2} \not \subset \operatorname{Supp}(D)$ or $\bar{L}_{5} \not \subset \operatorname{Supp}(D)$, which implies that either $a_{2} \leqslant 1$ or $a_{5} \leqslant 1$, respectively. 
Let us show that $L_{2}+L_{2}^{\prime}+L_{3} \sim-3 K_{X}$. We can easily see that

$$
\begin{aligned}
& \bar{L}_{2} \sim_{\mathbb{Q}} \pi^{*}\left(L_{2}\right)-\frac{5}{7} E_{1}-\frac{10}{7} E_{2}-\frac{8}{7} E_{3}-\frac{6}{7} E_{4}-\frac{4}{7} E_{5}-\frac{2}{7} E_{6}, \\
& \bar{L}_{2}^{\prime} \sim_{\mathbb{Q}} \pi^{*}\left(L_{2}^{\prime}\right)-\frac{5}{7} E_{1}-\frac{10}{7} E_{2}-\frac{8}{7} E_{3}-\frac{6}{7} E_{4}-\frac{4}{7} E_{5}-\frac{2}{7} E_{6}, \\
& \bar{L}_{3} \sim_{\mathbb{Q}} \pi^{*}\left(L_{3}\right)-\frac{4}{7} E_{1}-\frac{8}{7} E_{2}-\frac{12}{7} E_{3}-\frac{9}{7} E_{4}-\frac{6}{7} E_{5}-\frac{3}{7} E_{6},
\end{aligned}
$$

which implies that $L_{2}+L_{2}^{\prime}+L_{3} \sim_{\mathbb{Q}}-3 K_{X}$, since $\operatorname{Pic}(X) \cong \mathbb{Z}^{3}$ and

$$
L_{2} \cdot L_{2}=\frac{3}{7}, L_{2}^{\prime} \cdot L_{2}^{\prime}=\frac{3}{7}, L_{3} \cdot L_{3}=\frac{5}{7}, L_{2}^{\prime} \cdot L_{3}=\frac{8}{7}, L_{2} \cdot L_{3}=\frac{8}{7}, L_{2} \cdot L_{2}^{\prime}=\frac{10}{7},
$$

but $L_{2}+L_{2}^{\prime}+L_{3}$ is a Cartier divisor, which implies that $L_{2}+L_{2}^{\prime}+L_{3} \sim-3 K_{X}$.

Since c $\left(X, L_{2}+L_{2}^{\prime}+L_{3}\right)=1 / 4$, we may assume that $\operatorname{Supp}(D)$ does not contain at least one curve among $L_{2}, L_{2}^{\prime}$ and $L_{3}$ by Remark 2.1, which implies that either $a_{2} \leqslant 1$ or $a_{3} \leqslant 1$.

It follows from (4.5) and $a_{4} \leqslant 2$ that $\mu a_{i}<1$ for every $i$. By Lemma 4.6, there exists a point

$$
Q \in\left\{E_{2} \cap E_{3}, E_{3} \cap E_{4}, E_{4} \cap E_{5}\right\},
$$

such that (4.4) is not Kawamata $\log$ terminal at the point $Q \in \bar{X}$, but it is Kawamata log terminal elsewhere. Take $k \in\{2,3,4\}$ such that $Q=E_{k} \cap E_{k+1}$. It follows from Lemma 2.3 that

$$
\left\{\begin{array}{l}
2 a_{k}-a_{k-1}-a_{k+1}=\bar{D} \cdot E_{k} \geqslant \operatorname{mult}_{Q}\left(\bar{D} \cdot E_{k}\right)>\frac{1}{\mu}-a_{k+1}>\frac{3}{2}-a_{k+1}, \\
2 a_{k+1}-a_{k}-a_{k+2}=\bar{D} \cdot E_{k+1} \geqslant \operatorname{mult}_{Q}\left(\bar{D} \cdot E_{k+1}\right)>\frac{1}{\mu}-a_{k} \geqslant \frac{3}{2}-a_{k},
\end{array}\right.
$$

which is impossible by (4.5), since $a_{4} \leqslant 1$, and either $a_{2} \leqslant 1$ or $a_{3} \leqslant 1$.

Lemma 4.12. Suppose that $m=7$. Then the following conditions are equivalent:

- the curve $R$ is irreducible,

- the surface $\bar{X}$ contains an irreducible curve $\bar{L}_{4}$ such that $\bar{L}_{4} \cdot \bar{L}_{4}=-1$ and $\bar{L}_{4} \cdot E_{4}=1$.

- the surface $\bar{X}$ contains an irreducible curve $\bar{L}_{4}$ such that $\bar{L}_{4} \cdot \bar{L}_{4}=-1, \bar{L}_{4} \cdot E_{4}=1$ and

$$
\omega \circ \pi\left(\bar{L}_{4}\right) \subset \operatorname{Supp}(R) \text {. }
$$

Proof. Suppose that $\bar{X}$ has an irreducible curve $\bar{L}_{4}$ such that $\bar{L}_{4} \cdot \bar{L}_{4}=-1$ and $\bar{L}_{4} \cdot E_{4}=1$. Then

$$
\bar{L}_{4} \sim \mathbb{Q} \pi^{*}\left(L_{4}\right)-\frac{1}{2} E_{1}-E_{2}-\frac{3}{2} E_{3}-2 E_{4}-\frac{3}{2} E_{5}-E_{6}-\frac{1}{2} E_{7},
$$

where $L_{4}=\pi\left(\bar{L}_{4}\right)$. Then $\tau\left(\bar{L}_{4}\right)=\bar{L}_{4}$ and $\omega\left(L_{4}\right) \subset \operatorname{Supp}(R)$, because

$$
-1+\bar{L}_{4} \cdot \tau\left(\bar{L}_{4}\right)=\bar{L}_{4} \cdot\left(\bar{L}_{4}+\tau\left(\bar{L}_{4}\right)\right)=\bar{L}_{4} \cdot\left(\pi^{*}\left(-2 K_{X}\right)-E_{1}-2 E_{2}-3 E_{3}-4 E_{4}-3 E_{5}-2 E_{6}-E_{7}\right)=-2 .
$$

Suppose now that the curve $R$ is reducible. Let us show that the surface $\bar{X}$ contains an irreducible curve $\bar{L}_{4}$ such that $\bar{L}_{4} \cdot \bar{L}_{4}=-1$ and $\bar{L}_{4} \cdot E_{4}=1$.

Let $\eta: \bar{X} \rightarrow \bar{X}^{\prime}$ be a contraction of the curve $\bar{C}$. Then there is a commutative diagram

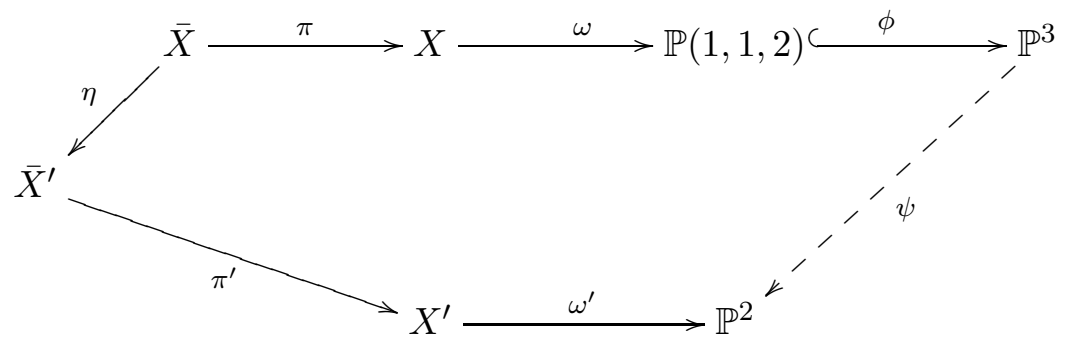


where $\pi^{\prime}$ is a minimal resolution, $\phi$ is an anticanonical embedding, $\psi$ is a projection from $\phi \circ \omega(P)$, and $\omega^{\prime}$ is a double cover branched at $\psi \circ \phi(R)$. Note that $X^{\prime}$ is a del Pezzo surface and $K_{X^{\prime}}^{2}=2$.

The morphism $\pi^{\prime}$ contracts the smooth curves $\eta\left(E_{2}\right), \eta\left(E_{3}\right), \eta\left(E_{4}\right), \eta\left(E_{5}\right)$ and $\eta\left(E_{6}\right)$. But

$$
\eta\left(E_{2}\right) \in \operatorname{Sing}\left(X^{\prime}\right)
$$

and $X^{\prime}$ has a singularity of type $\mathbb{A}_{5}$ at the point $\eta\left(E_{2}\right)$. Put $P^{\prime}=\eta\left(E_{2}\right)$.

Put $R^{\prime}=\psi \circ \phi(R)$. Then $R^{\prime}$ is reducible, since $R$ is reducible.

Since $\operatorname{Sing}(\mathbb{P}(1,1,2)) \notin R$, one of the following cases hold:

- either $\phi(R)$ is a union of a smooth conic and an irreducible quartic,

- or the curve $\phi(R)$ is a union of three different smooth conics.

The case when the curve $\phi(R)$ consists of a union of three different smooth conics is impossible, since the surface $X^{\prime}$ has a singularity of type $\mathbb{A}_{5}$ at the point $P^{\prime}=\operatorname{Sing}\left(X^{\prime}\right)$.

We see that the curve $\phi(R)$ is a union of a smooth conic and an irreducible quartic curve, which easily implies that $R^{\prime}$ is a union of a line $L$ and an irreducible cubic curve $Z$. Then

$$
\operatorname{mult}_{\omega^{\prime}\left(P^{\prime}\right)}(L \cdot Z)=3 \text {, }
$$

because $X^{\prime}$ has a singularity of type $\mathbb{A}_{5}$ at the point $P^{\prime}$. Then $\bar{X}$ contains a curve $\bar{L}_{4}$ such that

$$
\omega^{\prime} \circ \pi^{\prime} \circ \eta\left(\bar{L}_{4}\right)=L,
$$

and $\bar{L}_{4}$ is irreducible. Then $\bar{L}_{4} \cdot \bar{L}_{4}=-1$ and $\bar{L}_{4} \cdot E_{4}=1$.

The proof of Lemma 4.12 can be simplified using the results obtained in [31, Section 2].

Lemma 4.13. Suppose that $m=7$ and $R$ is irreducible. Then $\mu \geqslant \operatorname{lct}_{3}(X)=3 / 5$.

Proof. Arguing as in the proofs of Lemmas 4.10 and 4.11, we see that there is an irreducible smooth rational curve $\bar{L}_{2}$ on the surface $\bar{X}$ such that $\bar{L}_{2} \cdot \bar{L}_{2}=-1$ and

$$
-K_{\bar{X}} \cdot \bar{L}_{2}=E_{2} \cdot \bar{L}_{2}=1 \text {, }
$$

which implies that $E_{1} \cdot \bar{L}_{2}=E_{3} \cdot \bar{L}_{2}=E_{4} \cdot \bar{L}_{2}=E_{5} \cdot \bar{L}_{2}=E_{6} \cdot \bar{L}_{2}=E_{7} \cdot \bar{L}_{2}=\bar{C} \cdot \bar{L}_{2}=0$.

Put $\bar{L}_{5}=\tau\left(\bar{L}_{2}\right)$. Then $\bar{L}_{5} \cdot \bar{L}_{5}=-1$ and $-K_{\bar{X}} \cdot \bar{L}_{5}=E_{5} \cdot \bar{L}_{5}=1$, which implies that

$$
E_{1} \cdot \bar{L}_{5}=E_{2} \cdot \bar{L}_{5}=E_{3} \cdot \bar{L}_{5}=E_{4} \cdot \bar{L}_{5}=E_{6} \cdot \bar{L}_{5}=E_{7} \cdot \bar{L}_{5}=\bar{C} \cdot \bar{L}_{5}=0 \text {. }
$$

Since the branch curve $R$ is reducible by Lemma 4.12, one can show that there exists an irreducible smooth rational curve $\bar{L}_{3}$ on the surface $\bar{X}$ such that $\bar{L}_{3} \cdot \bar{L}_{3}=-1$ and

$$
-K_{\bar{X}} \cdot \bar{L}_{3}=E_{3} \cdot \bar{L}_{3}=1 \text {, }
$$

which implies that $E_{1} \cdot \bar{L}_{3}=E_{2} \cdot \bar{L}_{3}=E_{4} \cdot \bar{L}_{3}=E_{5} \cdot \bar{L}_{3}=E_{6} \cdot \bar{L}_{3}=E_{7} \cdot \bar{L}_{3}=\bar{C} \cdot \bar{L}_{3}=0$.

Put $\bar{L}_{6}=\tau\left(\bar{L}_{2}\right), \bar{L}_{5}=\tau\left(\bar{L}_{3}\right), L_{2}=\pi\left(\bar{L}_{2}\right), L_{3}=\pi\left(\bar{L}_{4}\right), L_{5}=\pi\left(\bar{L}_{5}\right)$ and $L_{6}=\pi\left(\bar{L}_{6}\right)$. Then

$$
\begin{gathered}
\bar{L}_{2} \sim_{\mathbb{Q}} \pi^{*}\left(L_{2}\right)-\frac{3}{4} E_{1}-\frac{3}{2} E_{2}-\frac{5}{4} E_{3}-E_{4}-\frac{3}{4} E_{5}-\frac{1}{2} E_{6}-\frac{1}{4} E_{7}, \\
\bar{L}_{3} \sim_{\mathbb{Q}} \pi^{*}\left(L_{3}\right)-\frac{5}{8} E_{1}-\frac{5}{4} E_{2}-\frac{15}{8} E_{3}-\frac{3}{2} E_{4}-\frac{9}{8} E_{5}-\frac{3}{4} E_{6}-\frac{3}{8} E_{7}, \\
\bar{L}_{5} \sim_{\mathbb{Q}} \pi^{*}\left(L_{5}\right)-\frac{3}{8} E_{1}-\frac{3}{4} E_{2}-\frac{9}{8} E_{3}-\frac{3}{2} E_{4}-\frac{15}{8} E_{5}-\frac{5}{4} E_{6}-\frac{5}{8} E_{7}, \\
\bar{L}_{6} \sim_{\mathbb{Q}} \pi^{*}\left(L_{6}\right)-\frac{1}{4} E_{1}-\frac{1}{2} E_{2}-\frac{3}{4} E_{3}-E_{4}-\frac{5}{4} E_{5}-\frac{3}{2} E_{6}-\frac{3}{4} E_{7},
\end{gathered}
$$

which implies that $L_{2}+2 L_{3} \sim-3 K_{X}$. Indeed, we have $L_{2}+2 L_{3} \sim_{\mathbb{Q}}-3 K_{X}$, since

$$
L_{2} \cdot L_{2}=\frac{1}{2}, L_{3} \cdot L_{3}=\frac{7}{8}, L_{2} \cdot L_{3}=\frac{5}{4},
$$

and $\operatorname{Pic}(X) \cong \mathbb{Z}^{3}$. But $L_{2}+2 L_{3}$ is a Cartier divisor, which implies that $L_{2}+2 L_{3} \sim-3 K_{X}$.

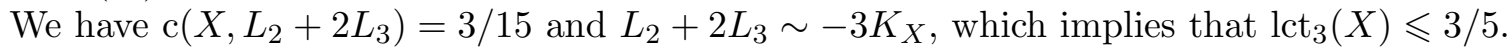


To complete the proof, it is enough to show that $\mu \geqslant 3 / 5$.

Suppose that $\mu<3 / 5$. Let us derive a contradiction.

By Remark 2.1, we may assume that the support of the divisor $\bar{D}$ does not contain at least one components of every curve $\bar{L}_{2}+\bar{L}_{6}, \bar{L}_{2}+2 \bar{L}_{3}, \bar{L}_{3}+\bar{L}_{5}$. But

$$
\bar{D} \cdot \bar{L}_{i}=1-a_{i}
$$

which implies that $a_{i} \leqslant 1$ if $\bar{L}_{i} \not \subset \operatorname{Supp}(\bar{D})$. Therefore, either $a_{3} \leqslant 1$ or $a_{2} \leqslant 1$ and $a_{5} \leqslant 1$.

If $a_{3} \leqslant 1$, then it follows from (4.5) that

$$
a_{1} \leqslant \frac{7}{8}, a_{2} \leqslant \frac{6}{5}, a_{3} \leqslant 1, a_{4} \leqslant \frac{4}{3}, a_{5} \leqslant \frac{5}{3}, a_{6} \leqslant \frac{3}{2}, a_{7} \leqslant \frac{7}{8} .
$$

If $a_{2} \leqslant 1$ and $a_{5} \leqslant 1$, then it follows from (4.5) that

$$
a_{1} \leqslant \frac{7}{8}, a_{2} \leqslant 1, a_{3} \leqslant \frac{3}{2}, a_{4} \leqslant \frac{4}{3}, a_{5} \leqslant 1, a_{6} \leqslant \frac{6}{5}, a_{7} \leqslant \frac{7}{8} .
$$

By Lemma 4.6, there exists $k \in\{2,3,4,5\}$ such that (4.4) is not Kawamata log terminal at the point $E_{k} \cap E_{k+1}$ and is Kawamata log terminal outside of $E_{k} \cap E_{k+1}$.

Put $Q=E_{k} \cap E_{k+1}$. Then it follows from Lemma 2.3 that

$$
\left\{\begin{array}{l}
2 a_{k}-a_{k-1}-a_{k+1}=\bar{D} \cdot E_{k} \geqslant \operatorname{mult}_{Q}\left(\bar{D} \cdot E_{k}\right)>\frac{1}{\mu}-a_{k+1}>\frac{5}{3}-a_{k+1}, \\
2 a_{k+1}-a_{k}-a_{k+2}=\bar{D} \cdot E_{k+1} \geqslant \operatorname{mult}_{Q}\left(\bar{D} \cdot E_{k+1}\right)>\frac{1}{\mu}-a_{k} \geqslant \frac{5}{3}-a_{k},
\end{array}\right.
$$

which is impossible by (4.5), since we assume that either $a_{3} \leqslant 1$ or $a_{2} \leqslant 1$ and $a_{5} \leqslant 1$.

Lemma 4.14. Suppose that $m=7$ and $R$ is reducible. Then $\mu \geqslant \operatorname{lct}_{2}(X)=1 / 2$.

Proof. By Lemma 4.12, the surface $X$ contains an irreducible curve $\bar{L}_{4}$ such that

$$
\omega \circ \pi\left(\bar{L}_{4}\right) \subset \operatorname{Supp}(R)
$$

and $-\bar{L}_{4} \cdot \bar{L}_{4}=\bar{L}_{4} \cdot E_{4}=1$. Then $-K_{\bar{X}} \cdot \bar{L}_{4}=1$, which implies that

$$
E_{1} \cdot \bar{L}_{4}=E_{2} \cdot \bar{L}_{4}=E_{3} \cdot \bar{L}_{4}=E_{5} \cdot \bar{L}_{4}=E_{6} \cdot \bar{L}_{4}=E_{7} \cdot \bar{L}_{4}=\bar{C} \cdot \bar{L}_{4}=0 .
$$

Put $L_{4}=\pi\left(\bar{L}_{4}\right)$. Then $2 L_{4} \sim-2 K_{X}$ and

$$
\bar{L}_{4} \sim_{\mathbb{Q}} \pi^{*}\left(L_{4}\right)-\frac{1}{2} E_{1}-E_{2}-\frac{3}{2} E_{3}-2 E_{4}-\frac{3}{2} E_{5}-E_{6}-\frac{1}{2} E_{7},
$$

which implies that $\operatorname{lct}_{2}(X) \leqslant \mathrm{c}\left(X, L_{4}\right)=1 / 2$.

To complete the proof, it is enough to show that $\mu \geqslant 1 / 2$.

Suppose that $\mu<1 / 2$. Let us derive a contradiction.

By Remark 2.1, we may assume that $L_{4} \not \subset \operatorname{Supp}(D)$. Then

$$
0 \leqslant \bar{L}_{4} \cdot \bar{D}=1-a_{4},
$$

which implies that $a_{4} \leqslant 1$. Thus, it follows from (4.5) that

$$
a_{1} \leqslant \frac{7}{8}, a_{2} \leqslant \frac{3}{2}, a_{3} \leqslant \frac{5}{4}, a_{4} \leqslant 1, a_{5} \leqslant \frac{5}{4}, a_{6} \leqslant \frac{3}{2}, a_{7} \leqslant \frac{7}{8} .
$$

It follows from Lemma 4.6 that there exists a point

$$
Q \in\left\{E_{2} \cap E_{3}, E_{3} \cap E_{4}, E_{4} \cap E_{5}, E_{5} \cap E_{6}\right\}
$$

such that $\operatorname{LCS}\left(\bar{X}, \mu \bar{D}+\sum_{i=1}^{7} \mu a_{i} E_{i}\right)=Q$.

Without loss of generality, we may assume that either $Q=E_{2} \cap E_{3}$ or $Q=E_{3} \cap E_{4}$.

If $Q=E_{3} \cap E_{4}$, then it follows from Lemma 2.3 that

$$
2 a_{4}-a_{3}-a_{5}=\bar{D} \cdot E_{4} \geqslant \operatorname{mult}_{Q}\left(\bar{D} \cdot E_{4}\right)>\frac{1}{\mu}-a_{3}>2-a_{3},
$$


which together with (4.5) imply that $a_{4}>1$, which is a contradiction.

If $Q=E_{2} \cap E_{3}$, then it follows from Lemma 2.3 that

$$
2 a_{3}-a_{2}-a_{4}=\bar{D} \cdot E_{3} \geqslant \operatorname{mult}_{Q}\left(\bar{D} \cdot E_{3}\right)>\frac{1}{\mu}-a_{2}>2-a_{2},
$$

which together with (4.5) immediately leads to a contradiction.

Lemma 4.15. Suppose that $m=8$. $\operatorname{Then}_{\mu} \geqslant \operatorname{lct}_{3}(X)=1 / 2$.

Proof. Arguing as in the proofs of Lemmas 4.10 and 4.11, we see that there is an irreducible smooth rational curve $\bar{L}_{3}$ on the surface $\bar{X}$ such that $\bar{L}_{3} \cdot \bar{L}_{3}=-1$ and

$$
-K_{\bar{X}} \cdot \bar{L}_{3}=E_{3} \cdot \bar{L}_{3}=1,
$$

which implies that $E_{1} \cdot \bar{L}_{3}=E_{2} \cdot \bar{L}_{3}=E_{4} \cdot \bar{L}_{3}=E_{5} \cdot \bar{L}_{3}=E_{6} \cdot \bar{L}_{3}=E_{7} \cdot \bar{L}_{3}=\bar{C} \cdot \bar{L}_{3}=0$.

Put $\bar{L}_{6}=\tau\left(\bar{L}_{3}\right)$. Then $\bar{L}_{6} \cdot \bar{L}_{6}=-1$ and $-K_{\bar{X}} \cdot \bar{L}_{6}=E_{6} \cdot \bar{L}_{6}=1$, which implies that

$$
E_{1} \cdot \bar{L}_{6}=E_{2} \cdot \bar{L}_{6}=E_{3} \cdot \bar{L}_{6}=E_{4} \cdot \bar{L}_{6}=E_{5} \cdot \bar{L}_{6}=E_{7} \cdot \bar{L}_{6}=\bar{C} \cdot \bar{L}_{6}=0 \text {. }
$$

Put $L_{3}=\pi\left(\bar{L}_{3}\right)$ and $L_{6}=\pi\left(\bar{L}_{6}\right)$. Then $3 L_{3} \sim 3 L_{6} \sim-3 K_{X}$. On the other hand, we have

$$
\begin{aligned}
& \bar{L}_{3} \sim_{\mathbb{Q}} \pi^{*}\left(L_{3}\right)-\frac{2}{3} E_{1}-\frac{4}{3} E_{2}-2 E_{3}-\frac{5}{3} E_{4}-\frac{4}{3} E_{5}-E_{6}-\frac{2}{3} E_{7}-\frac{1}{3} E_{8}, \\
& \bar{L}_{6} \sim_{\mathbb{Q}} \pi^{*}\left(L_{6}\right)-\frac{1}{3} E_{1}-\frac{2}{3} E_{2}-E_{3}-\frac{4}{3} E_{4}-\frac{5}{3} E_{5}-2 E_{6}-\frac{4}{3} E_{7}-\frac{2}{3} E_{8},
\end{aligned}
$$

which implies $\mathrm{c}\left(X, L_{3}\right)=\mathrm{c}\left(X, L_{6}\right)=1 / 2$. $\operatorname{Then} \operatorname{lct}_{3}(X) \leqslant 1 / 2$.

To complete the proof, it is enough to show that $\mu \geqslant 1 / 2$.

Suppose that $\mu<1 / 2$. Let us derive a contradiction.

By Remark 2.1, we may assume that $\operatorname{Supp}(\bar{D})$ does not contain $\bar{L}_{3}$ and $\bar{L}_{6}$. Then

$$
1-a_{3}=\bar{D} \cdot \bar{L}_{3} \geqslant 0
$$

which implies that $a_{3} \leqslant 1$. Similarly, we have $a_{6} \leqslant 1$. Then it follows from (4.5) that

$$
a_{1} \leqslant \frac{8}{9}, a_{2} \leqslant \frac{7}{6}, a_{3} \leqslant 1, a_{4} \leqslant \frac{4}{3}, a_{5} \leqslant \frac{4}{3}, a_{6} \leqslant 1, a_{7} \leqslant \frac{7}{6}, a_{8} \leqslant \frac{8}{9} .
$$

By Lemma 4.6, there exists $k \in\{2,3,4,5,6\}$ such that (4.4) is not Kawamata log terminal at the point $E_{k} \cap E_{k+1}$ and is Kawamata log terminal outside of the point $E_{k} \cap E_{k+1}$.

Put $Q=E_{k} \cap E_{k+1}$. Then it follows from Lemma 2.3 that

$$
\left\{\begin{array}{l}
2 a_{k}-a_{k-1}-a_{k+1}=\bar{D} \cdot E_{k} \geqslant \operatorname{mult}_{Q}\left(\bar{D} \cdot E_{k}\right)>\frac{1}{\mu}-a_{k+1}>\frac{1}{2}-a_{k+1}, \\
2 a_{k+1}-a_{k}-a_{k+2}=\bar{D} \cdot E_{k+1} \geqslant \operatorname{mult}_{Q}\left(\bar{D} \cdot E_{k+1}\right)>\frac{1}{\mu}-a_{k} \geqslant \frac{1}{2}-a_{k},
\end{array}\right.
$$

which is impossible by (4.5), since $a_{3} \leqslant 1$ and $a_{6} \leqslant 1$.

The assertion of Theorem 4.1 is proved.

\section{ONE NON-CYCLIC SINGULAR POINT}

Let $X$ be a sextic surface in $\mathbb{P}(1,1,2,3)$ with canonical singularities such that $|\operatorname{Sing}(X)|=1$, and $\operatorname{Sing}(X)$ consists of a singular point of type $\mathbb{D}_{4}, \mathbb{D}_{5}, \mathbb{D}_{6}, \mathbb{D}_{7}, \mathbb{D}_{8}, \mathbb{E}_{6}, \mathbb{E}_{7}$ or $\mathbb{E}_{8}$.

Theorem 5.1. The following equality holds:

$$
\operatorname{lct}(X)=\left\{\begin{array}{l}
\operatorname{lct}_{2}(X)=1 / 3 \text { if } P \text { is a point of type } \mathbb{D}_{8}, \\
\operatorname{lct}_{2}(X)=2 / 5 \text { if } P \text { is a point of type } \mathbb{D}_{7}, \\
\operatorname{lct}_{1}(X) \text { in the remaining cases. }
\end{array}\right.
$$


Corollary 5.2. The inequality $\operatorname{lct}(X) \leqslant 1 / 2$ holds.

In the rest of this section we will prove Theorem 5.1 .

Let $D$ be an effective $\mathbb{Q}$-divisor on $X$ such that $D \sim_{\mathbb{Q}}-K_{X}$. We must show that

$$
\mathrm{c}(X, D) \geqslant\left\{\begin{array}{l}
\operatorname{lct}_{2}(X)=1 / 3 \text { if } P \text { is a point of type } \mathbb{D}_{8}, \\
\operatorname{lct}_{2}(X)=2 / 5 \text { if } P \text { is a point of type } \mathbb{D}_{7}, \\
\operatorname{lct}_{1}(X) \text { in the remaining cases. }
\end{array}\right.
$$

to prove Theorem 5.1. Put $\mu=\mathrm{c}(X, D)$.

Suppose that $\mu<\operatorname{lct}_{1}(X)$. Then $\operatorname{LCS}(X, \mu D)=\operatorname{Sing}(X)$ by Lemma 2.6, Put $P=\operatorname{Sing}(X)$.

Let $\pi: \bar{X} \rightarrow X$ be a minimal resolution, let $E_{1}, E_{2} \ldots, E_{m}$ be irreducible $\pi$-exceptional curves, let $C$ be the curve in $\left|-K_{X}\right|$ such that $P \in C$, and let $\bar{C}$ be its proper transform on $\bar{X}$. Then

$$
\bar{C} \sim_{\mathbb{Q}} \pi^{*}(C)-\sum_{i=1}^{m} n_{i} E_{i},
$$

where $n_{i} \in \mathbb{N}$. Without loss of generality, we may assume that $E_{3} \cdot \sum_{i \neq 3} E_{i}=3$. Then

$$
\operatorname{lct}_{1}(X)=c(X, C)=\frac{1}{n_{3}}=\left\{\begin{array}{l}
1 / 2 \text { if } P \text { is of type } \mathbb{D}_{4}, \mathbb{D}_{5}, \mathbb{D}_{6}, \mathbb{D}_{7} \text { or } \mathbb{D}_{8}, \\
1 / 3 \text { if } P \text { is of type } \mathbb{E}_{6}, \\
1 / 4 \text { if } P \text { is of type } \mathbb{E}_{7} \\
1 / 6 \text { if } P \text { is of type } \mathbb{E}_{8}
\end{array}\right.
$$

By Remark 2.1, we may assume that $C \not \subset \operatorname{Supp}(D)$, since the curve $C$ is irreducible.

Let $\bar{D}$ be the proper transform of the divisor $D$ on the surface $\bar{X}$. Then

$$
\bar{D} \sim_{\mathbb{Q}} \pi^{*}(D)-\sum_{i=1}^{m} a_{i} E_{i},
$$

where $a_{i}$ is a non-negative rational number. Then

$$
K_{\bar{X}}+\mu\left(\bar{D}+\sum_{i=1}^{m} a_{i} E_{i}\right) \sim_{\mathbb{Q}} \pi^{*}\left(K_{X}+\mu D\right),
$$

which implies that $\left(\bar{X}, \mu \bar{D}+\sum_{i=1}^{m} \mu a_{i} E_{i}\right)$ is not Kawamata log terminal (see Remark 2.4).

Lemma 5.3. The equality $\mu a_{3}=1$ holds.

Proof. The equality $\mu a_{3}=1$ follows from Lemma 2.5.

Lemma 5.4. Suppose that $P$ is not a point of type $\mathbb{E}_{6}, \mathbb{E}_{7}$ or $\mathbb{E}_{8}$. Then

$$
\mu \geqslant\left\{\begin{array}{l}
\operatorname{lct}_{2}(X)=1 / 3 \text { if } P \text { is a point of type } \mathbb{D}_{8}, \\
\operatorname{lct}_{2}(X)=2 / 5 \text { if } P \text { is a point of type } \mathbb{D}_{7},
\end{array}\right.
$$

and $P$ is either a point of type $\mathbb{D}_{7}$ or is a point of type $\mathbb{D}_{8}$.

Proof. Without loss of generality, we may assume that the diagram

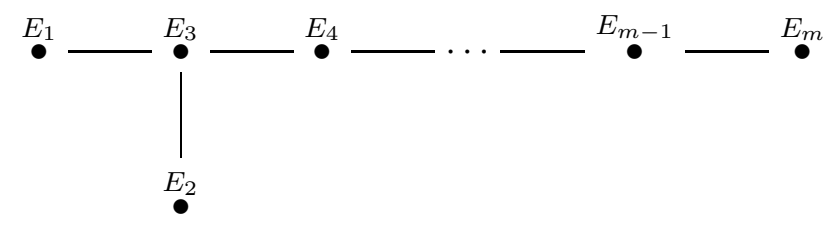


shows how the $\pi$-exceptional curves intersect each other. Then

$$
\bar{C} \sim_{\mathbb{Q}} \pi^{*}(C)-E_{1}-E_{2}-E_{m}-\sum_{i=3}^{m-1} 2 E_{i},
$$

which implies that $\bar{C} \cdot E_{m-1}=1$ and $\bar{C} \cdot E_{i}=0 \Longleftrightarrow i \neq m-1$. Then

$$
\left\{\begin{array}{l}
1-a_{m-1}=\bar{D} \cdot \bar{C} \geqslant 0 \\
2 a_{1}-a_{3}=\bar{D} \cdot E_{1} \geqslant 0 \\
2 a_{2}-a_{3}=\bar{D} \cdot E_{2} \geqslant 0 \\
2 a_{3}-a_{1}-a_{2}-a_{3}=\bar{D} \cdot E_{3} \geqslant 0 \\
\cdots \\
2 a_{m-1}-a_{m-2}-a_{m}=\bar{D} \cdot E_{m-1} \geqslant 0 \\
2 a_{m}-a_{m-1}=\bar{D} \cdot E_{m} \geqslant 0
\end{array}\right.
$$

which easily implies that $a_{3} \leqslant 2$ if $m \leqslant 6$. But $\mu a_{3}=1$ and $\mu<\operatorname{lct}_{1}(X)=1 / 2$ by Lemma 5.3 , which implies that either $m=7$ or $m=8$.

Arguing as in the proofs of Lemmas 4.10 and 4.11, we may assume that there is an irreducible smooth rational curve $\bar{L}_{1}$ on the surface $\bar{X}$ such that $\bar{L}_{1} \cdot \bar{L}_{1}=-1$ and

$$
-K_{\bar{X}} \cdot \bar{L}_{1}=E_{1} \cdot \bar{L}_{1}=1,
$$

which implies that $\bar{C} \cdot \bar{L}_{1}=0$ and $E_{i} \cdot \bar{L}_{1}=0 \Longleftrightarrow i \neq 1$.

Let $\omega: X \rightarrow \mathbb{P}(1,1,2)$ be the natural double cover given by $\left|-2 K_{X}\right|$, and let $\tau$ be a biregular involution of the surface $\bar{X}$ that is induced by $\omega$. Put $\bar{L}_{2}=\tau\left(\bar{L}_{1}\right)$. If $m=7$, then

$$
-K_{\bar{X}} \cdot \bar{L}_{2}=E_{2} \cdot \bar{L}_{2}=1
$$

and $\bar{L}_{2} \cdot \bar{L}_{2}=-1$, which implies that $\bar{C} \cdot \bar{L}_{2}=0$ and $E_{i} \cdot \bar{L}_{2}=0 \Longleftrightarrow i \neq 2$.

Put $L_{1}=\pi\left(\bar{L}_{1}\right)$ and $L_{2}=\pi\left(\bar{L}_{2}\right)$. Then $L_{1}+L_{2} \sim-2 K_{X}$. If $m=7$, then

$$
\begin{aligned}
& \bar{L}_{1} \sim_{\mathbb{Q}} \pi^{*}\left(L_{1}\right)-\frac{7}{4} E_{1}-\frac{5}{4} E_{2}-\frac{5}{2} E_{3}-2 E_{4}-\frac{3}{2} E_{5}-E_{6}-\frac{1}{2} E_{7}, \\
& \bar{L}_{2} \sim_{\mathbb{Q}} \pi^{*}\left(L_{2}\right)-\frac{5}{4} E_{1}-\frac{7}{4} E_{2}-\frac{5}{2} E_{3}-2 E_{4}-\frac{3}{2} E_{5}-E_{6}-\frac{1}{2} E_{7},
\end{aligned}
$$

which implies that $\mathrm{c}\left(X, L_{1}+L_{2}\right)=1 / 5$ and $\operatorname{lct}_{2}(X) \leqslant 2 / 5$. If $m=7$, then

$$
a_{3} \leqslant \frac{5}{2}
$$

by (5.5). But $\mu a_{3}=1$ by Lemma 5.3. Then $\mu \geqslant 2 / 5$ if $m=7$, which is exactly what we need.

We may assume that $m=8$. Then $\bar{L}_{2}=\bar{L}_{1}$ and

$$
\bar{L}_{1} \sim_{\mathbb{Q}} \pi^{*}\left(L_{1}\right)-2 E_{1}-\frac{3}{2} E_{2}-3 E_{3}-\frac{5}{2} E_{4}-2 E_{5}-\frac{3}{2} E_{6}-E_{7}-\frac{1}{2} E_{8},
$$

which implies that $\operatorname{lct}_{2}(X) \leqslant \mathrm{c}\left(X, L_{1}\right)=1 / 3$. But $a_{3} \leqslant 1 / 3$ by (5.5) and $\mu a_{3}=1$ by Lemma 5.3 ,

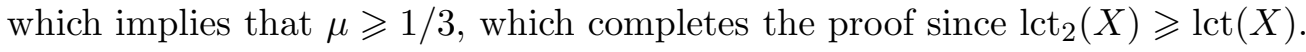

To complete the proof of Theorem [5.1, we may assume that $P$ is a point of type $\mathbb{E}_{6}, \mathbb{E}_{7}$ or $\mathbb{E}_{8}$. Without loss of generality, we may assume that the diagram

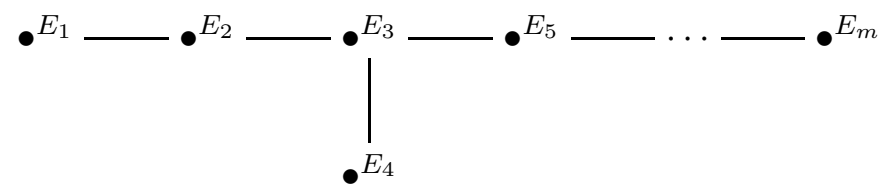

shows how the $\pi$-exceptional curves intersect each other. It is well-known (cf. [29] [30]) that 
- if $m=6$, then $\bar{C} \cdot E_{4}=1$, which implies that and $\bar{C} \cdot E_{i}=0 \Longleftrightarrow i \neq 4$,

- if $m=7$, then $\bar{C} \cdot E_{1}=1$, which implies that and $\bar{C} \cdot E_{i}=0 \Longleftrightarrow i \neq 1$,

- if $m=8$, then $\bar{C} \cdot E_{8}=1$, which implies that and $\bar{C} \cdot E_{i}=0 \Longleftrightarrow i \neq 8$.

Put $k=4$ if $m=6$, put $k=1$ if $m=7$, put $k=8$ if $m=8$. Then

$$
\left\{\begin{array}{l}
1-a_{k}=\bar{D} \cdot \bar{C} \geqslant 0 \\
2 a_{1}-a_{3}=\bar{D} \cdot E_{1} \geqslant 0 \\
2 a_{2}-a_{3}-a_{1}=\bar{D} \cdot E_{2} \geqslant 0 \\
2 a_{3}-a_{2}-a_{4}-a_{5}=\bar{D} \cdot E_{3} \geqslant 0 \\
2 a_{4}-a_{3}=\bar{D} \cdot E_{4} \geqslant 0 \\
2 a_{5}-a_{3}-a_{6}=\bar{D} \cdot E_{5} \geqslant 0 \\
\cdots \\
2 a_{m-1}-a_{m-2}-a_{m}=\bar{D} \cdot E_{m-1} \geqslant 0 \\
2 a_{m}-a_{m-1}=\bar{D} \cdot E_{m} \geqslant 0
\end{array}\right.
$$

which implies that $a_{3}<n_{3}$. But $n_{3}=1 / \operatorname{lct}_{1}(X)$ and $\mu a_{3}=1$ by $\operatorname{Lemma}_{5.3}$. Then $\mu \geqslant \operatorname{lct}_{1}(X)$.

The assertion of Theorem 5.1 is proved.

\section{MANY SINGUlar POINTS}

Let $X$ be a sextic surface in $\mathbb{P}(1,1,2,3)$ with canonical singularities such that $|\operatorname{Sing}(X)| \geqslant 2$.

Theorem 6.1. The following equality holds:

$$
\operatorname{lct}(X)=\left\{\begin{array}{l}
\operatorname{lct}_{2}(X)=1 / 2 \text { if } \operatorname{Sing}(X) \text { consists of a point of type } \mathbb{A}_{7} \text { and a point of type } \mathbb{A}_{1}, \\
\operatorname{lct}_{2}(X)=2 / 3 \text { if } X \text { has a singular point of type } \mathbb{A}_{6}, \\
\operatorname{lct}_{2}(X)=2 / 3 \text { if } X \text { has a singular point of type } \mathbb{A}_{5}, \\
\operatorname{lct}_{2}(X)=\min \left(\operatorname{lct}_{1}(X), 4 / 5\right) \text { if } X \text { has a singular point of type } \mathbb{A}_{4}, \\
\operatorname{lct}_{1}(X) \text { in the remaining cases, }
\end{array}\right.
$$

and if there exists an effective $\mathbb{Q}$-divisor $D$ on the surface $X$ such that $D \sim_{\mathbb{Q}}-K_{X}$ and

$$
\mathrm{c}(X, D)=\operatorname{lct}(X)=\frac{2}{3},
$$

then either $D$ is an irreducible curve in $\left|-K_{X}\right|$ with a cusp at a point in $\operatorname{Sing}(X)$ of type $\mathbb{A}_{2}$, or the divisor $D$ is uniquely defined and it can be explicitly described.

Let $D$ be an arbitrary effective $\mathbb{Q}$-divisor on the surface $X$ such that

$$
D \sim_{\mathbb{Q}}-K_{X},
$$

and put $\mu=\mathrm{c}(X, D)$. To prove Theorem 6.1, it is enough to show that

$$
\mu \geqslant\left\{\begin{array}{l}
\operatorname{lct}_{2}(X)=1 / 2 \text { if } \operatorname{Sing}(X) \text { consists of a point of type } \mathbb{A}_{7} \text { and a point of type } \mathbb{A}_{1}, \\
\operatorname{lct}_{2}(X)=2 / 3 \text { if } X \text { has a singular point of type } \mathbb{A}_{6}, \\
\operatorname{lct}_{2}(X)=2 / 3 \text { if } X \text { has a singular point of type } \mathbb{A}_{5}, \\
\operatorname{lct}_{2}(X)=\min \left(\operatorname{lct}_{1}(X), 4 / 5\right) \text { if } X \text { has a singular point of type } \mathbb{A}_{4}, \\
\operatorname{lct}_{1}(X) \text { in the remaining cases, }
\end{array}\right.
$$

and if $\mu=\operatorname{lct}(X)=2 / 3$, then we have the following two possibilities:

- either $D$ is a curve in $\left|-K_{X}\right|$ with a cusp at a point in $\operatorname{Sing}(X)$ of type $\mathbb{A}_{2}$,

- or the divisor $D$ is uniquely defined and it can be explicitly described. 
Lemma 6.2. If $\operatorname{Sing}(X)$ has a point of type $\mathbb{D}_{4}, \mathbb{D}_{5}, \mathbb{D}_{6}, \mathbb{E}_{6}, \mathbb{E}_{7}$ or $\mathbb{E}_{8}$, then $\mu \geqslant \operatorname{lct}_{1}(X)$.

Proof. Suppose that $\operatorname{Sing}(X)$ has a point of type $\mathbb{D}_{4}, \mathbb{D}_{5}, \mathbb{D}_{6}, \mathbb{E}_{6}, \mathbb{E}_{7}$ or $\mathbb{E}_{8}$, but $\mu<\operatorname{lct}_{1}(X)$. Then

$$
\operatorname{LCS}(X, \mu D) \subsetneq \operatorname{Sing}(X)
$$

and $\operatorname{LCS}(X, \mu D)$ consists of a point in $\operatorname{Sing}(X)$ that is not of type $\mathbb{A}_{1}$ or $\mathbb{A}_{2}$ by Lemma 2.6.

If the locus $\operatorname{LCS}(X, \mu D)$ is a singular point of the surface $X$ of type $\mathbb{D}_{4}, \mathbb{D}_{5}, \mathbb{D}_{6}, \mathbb{E}_{6}, \mathbb{E}_{7}$ or $\mathbb{E}_{8}$, then arguing as in the proof of Theorem 5.1, we immediately obtain a contradiction.

By Remark 1.22, the locus $\operatorname{LCS}(X, \mu D)$ must be a singular point of the surface $X$ of type $\mathbb{A}_{3}$, and we can easily obtain a contradiction arguing as in the proof of Corollary 4.7.

Lemma 6.3. Suppose that $\operatorname{Sing}(X)$ consists of points of type $\mathbb{A}_{1}, \mathbb{A}_{2}$ or $\mathbb{A}_{3}$. Then $\mu \geqslant \operatorname{lct}(X)$. If

$$
\mu=\operatorname{lct}_{1}(X)=\frac{2}{3}
$$

then $D$ is a curve in $\left|-K_{X}\right|$ with a cusp at a point in $\operatorname{Sing}(X)$ of type $\mathbb{A}_{2}$.

Proof. This follows from Lemma 2.6 and the proof of Corollary 4.7.

By Remark 1.22 and Lemmas 6.2 and 6.2, we may assume that

$$
\operatorname{Sing}(X) \in\left\{\begin{array}{l}
\mathbb{A}_{7}+\mathbb{A}_{1}, \mathbb{A}_{6}+\mathbb{A}_{1}, \mathbb{A}_{5}+\mathbb{A}_{1}, \mathbb{A}_{5}+\mathbb{A}_{1}+\mathbb{A}_{1}, \mathbb{A}_{5}+\mathbb{A}_{2}, \mathbb{A}_{5}+\mathbb{A}_{2}+\mathbb{A}_{1}, \\
\mathbb{A}_{4}+\mathbb{A}_{4}, \mathbb{A}_{4}+\mathbb{A}_{3}, \mathbb{A}_{4}+\mathbb{A}_{2}+\mathbb{A}_{1}, \mathbb{A}_{4}+\mathbb{A}_{2}, \mathbb{A}_{4}+\mathbb{A}_{1}+\mathbb{A}_{1}, \mathbb{A}_{4}+\mathbb{A}_{1},
\end{array}\right\}
$$

which implies that there is a point $P \in \operatorname{Sing}(X)$ that is a point of type $\mathbb{A}_{m}$ for $m \in\{4,5,6,7\}$.

Let $\pi: \bar{X} \rightarrow X$ be a minimal resolution, let $E_{1}, E_{2}, \ldots, E_{m}$ be $\pi$-exceptional curves such that

$$
E_{i} \cdot E_{j} \neq 0 \Longleftrightarrow|i-j| \leqslant 1
$$

and $\pi\left(E_{i}\right)=P$ for every $i \in\{1, \ldots, m\}$, let $C$ be the unique curve in $\left|-K_{X}\right|$ such that $P \in C$, and let $\bar{C}$ be the proper transform of the curve $C$ on the surface $\bar{X}$. Then

$$
\bar{C} \cdot E_{1}=\bar{C} \cdot E_{m}=1,
$$

and $\bar{C} \cdot E_{2}=\bar{C} \cdot E_{3}=\cdots=\bar{C} \cdot E_{m-1}=0$. Note that $\bar{C} \cong \mathbb{P}^{1}$ and $\bar{C} \cdot \bar{C}=-1$.

Let $\bar{D}$ be the proper transform of $D$ on the surface $\bar{X}$. Then

$$
\bar{D} \sim_{\mathbb{Q}} \pi^{*}(D)-\sum_{i=1}^{m} a_{i} E_{i},
$$

where $a_{i}$ is a non-negative rational number. Then

$$
\left\{\begin{array}{l}
1-a_{1}-a_{m}=\bar{D} \cdot \bar{C} \geqslant 0 \\
2 a_{1}-a_{2}=\bar{D} \cdot E_{1} \geqslant 0 \\
\cdots \\
2 a_{m-1}-a_{m-2}-a_{m}=\bar{D} \cdot E_{m-1} \geqslant 0 \\
2 a_{m}-a_{m-1}=\bar{D} \cdot E_{m} \geqslant 0
\end{array}\right.
$$

Let $\eta: \bar{X} \rightarrow \bar{X}^{\prime}$ be a contraction of the curve $\bar{C}$. Then there is a commutative diagram

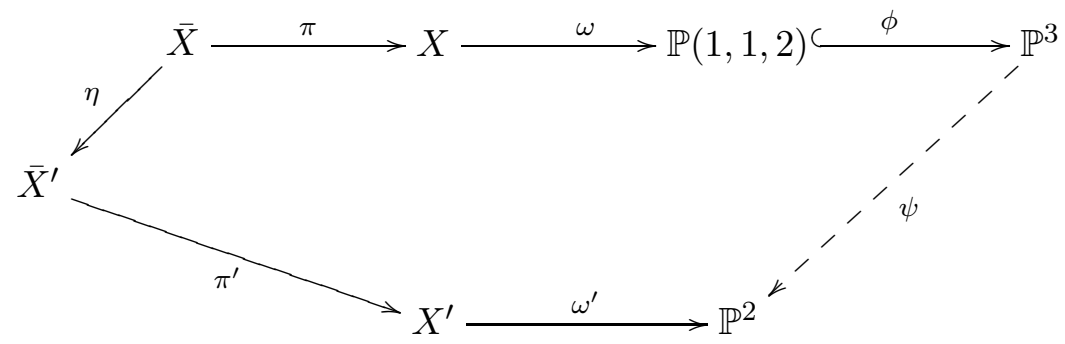


where $\omega$ and $\omega^{\prime}$ are natural double covers $\pi^{\prime}$ is a minimal resolution, $\phi$ is an anticanonical embedding, and $\psi$ is a projection from $\phi \circ \omega(P)$. Put $P^{\prime}=\eta\left(E_{2}\right)$. Then $P^{\prime} \in \operatorname{Sing}\left(X^{\prime}\right)$.

Remark 6.5. The birational morphism $\pi^{\prime}$ contracts the smooth curves $\eta\left(E_{2}\right), \eta\left(E_{3}\right), \ldots, \eta\left(E_{m-1}\right)$, and $\pi^{\prime} \circ \eta$ contracts all $\pi$-exceptional curves that are different from the curves $E_{1}, E_{2}, \ldots, E_{m}$.

Let $R$ be the branch curve in $\mathbb{P}(1,1,2)$ of the double cover $\omega$. Put $R^{\prime}=\psi \circ \phi(R)$.

Lemma 6.6. Suppose that $m=7$. Then $\mu \geqslant \operatorname{lct}_{2}(X)=1 / 2$.

Proof. Let $\alpha: \bar{X} \rightarrow \breve{X}$ be a contraction of the irreducible curves $\bar{C}, E_{7}, E_{6}, E_{5}, E_{4}, E_{3}$ and $E_{2}$, and let $F$ be the $\pi$-exceptional curve such that $\pi(F)$ is a point of type $\mathbb{A}_{1}$. Then

$$
\breve{X} \cong \mathbb{P}\left(\mathcal{O}_{\mathbb{P}^{1}} \oplus \mathcal{O}_{\mathbb{P}^{1}}(2)\right)
$$

Let $\breve{L}_{2}$ be the fiber of the projection $\breve{X} \rightarrow \mathbb{P}^{1}$ such that $\alpha(\bar{C}) \in \breve{L}_{2}$, and let $\bar{L}_{2}$ be the proper transform of the curve $\breve{L}_{2}$ on the surface $\bar{X}$ via $\alpha$. Then $\bar{L}_{2} \cdot \bar{L}_{2}=-1$ and

$$
-K_{\bar{X}} \cdot \bar{L}_{2}=E_{2} \cdot \bar{L}_{2}=F \cdot \bar{L}_{2}=1 \text {, }
$$

which implies that $E_{1} \cdot \bar{L}_{2}=E_{3} \cdot \bar{L}_{2}=E_{4} \cdot \bar{L}_{2}=E_{5} \cdot \bar{L}_{2}=E_{6} \cdot \bar{L}_{2}=E_{7} \cdot \bar{L}_{2}=\bar{C} \cdot \bar{L}_{2}=0$.

Let $\beta: \bar{X} \rightarrow \check{X}$ be a contraction of the curves $\bar{L}_{2}, E_{2}, \bar{C}, E_{7}, E_{6}, E_{5}, E_{4}$. Then

$$
\beta\left(E_{3}\right) \cdot \beta\left(E_{3}\right)=\beta(F) \cdot \beta(F)=0,
$$

and $\check{X}$ is a smooth del Pezzo surface such that $K_{\check{X}}^{2}=8$. Then $\check{X} \cong \mathbb{P}^{1} \times \mathbb{P}^{1}$.

Let $\check{L}_{4}$ be the curve in $|\beta(F)|$ such that $\beta\left(E_{4}\right) \in \check{L}_{4}$, and let $\bar{L}_{3}$ be its proper transform on the surface $\bar{X}$ via $\beta$. Then one can easily check that $\bar{L}_{4} \cdot \bar{L}_{4}=-1$ and

$$
-K_{\bar{X}} \cdot \bar{L}_{4}=E_{4} \cdot \bar{L}_{4}=1,
$$

which implies that $E_{1} \cdot \bar{L}_{4}=E_{2} \cdot \bar{L}_{4}=E_{3} \cdot \bar{L}_{4}=E_{5} \cdot \bar{L}_{4}=E_{6} \cdot \bar{L}_{4}=E_{7} \cdot \bar{L}_{4}=\bar{C} \cdot \bar{L}_{4}=F \cdot \bar{L}_{4}=0$.

Put $L_{4}=\pi\left(\bar{L}_{4}\right)$. Then one can easily check that

$$
\bar{L}_{4} \sim_{\mathbb{Q}} \pi^{*}\left(L_{4}\right)-\frac{1}{2} E_{1}-E_{2}-\frac{3}{2} E_{3}-2 E_{4}-\frac{3}{2} E_{5}-E_{6}-\frac{1}{2} E_{7},
$$

which implies that $\mathrm{c}\left(X, L_{4}\right)=1 / 2$. But $2 L_{4} \sim-2 K_{X}$, which implies that $\operatorname{lct}_{2}(X) \leqslant 1 / 2$.

Arguing as in the proof of Lemma 4.12, we see that $\omega\left(L_{4}\right) \subset \operatorname{Supp}(R)$.

Arguing as in the proof of Lemma 4.14 and using (6.4), we see that $\mu \geqslant \operatorname{lct}_{2}(X)=1 / 2$.

Lemma 6.7. Suppose that $m=6$. Then $\mu \geqslant \operatorname{lct}_{2}(X)=2 / 3$, and if $\mu=2 / 3$, then

- either $D$ is a curve in $\left|-K_{X}\right|$ with a cusp at a point in $\operatorname{Sing}(X)$ of type $\mathbb{A}_{2}$,

- or the divisor $D$ is uniquely defined and can be explicitly described.

Proof. Let $\alpha: \bar{X} \rightarrow \breve{X}$ be a contraction of the curves $\bar{C}, E_{6}, E_{5}, E_{4}, E_{3}, E_{2}$. Then $\breve{X}$ is a smooth surface such that $K_{\breve{X}}^{2}=7$, and $-K_{X}$ is nef. There is a birational morphism $\gamma: \breve{X} \rightarrow \hat{X}$ such that

$$
\hat{X} \cong \mathbb{P}\left(\mathcal{O}_{\mathbb{P}^{1}} \oplus \mathcal{O}_{\mathbb{P}^{1}}(2)\right)
$$

and $\gamma$ is a blow down of a smooth irreducible rational curve that does not contain the point $\alpha(\bar{C})$.

Let $\hat{L}_{2}$ be the fiber of the projection $\hat{X} \rightarrow \mathbb{P}^{1}$ such that $\gamma \circ \alpha(\bar{C}) \in \hat{L}_{2}$, and let $\bar{L}_{2}$ be the proper transform of the curve $\hat{L}_{2}$ on the surface $\bar{X}$ via $\gamma \circ \alpha$. Then $\bar{L}_{2} \cdot \bar{L}_{2}=-1$ and

$$
-K_{\bar{X}} \cdot \bar{L}_{2}=E_{2} \cdot \bar{L}_{2}=1
$$

which implies that $E_{1} \cdot \bar{L}_{2}=E_{3} \cdot \bar{L}_{2}=E_{4} \cdot \bar{L}_{2}=E_{5} \cdot \bar{L}_{2}=E_{6} \cdot \bar{L}_{2}=\bar{C} \cdot \bar{L}_{2}=0$.

Let $\beta: \bar{X} \rightarrow \check{X}$ be a contraction of the curves $\bar{L}_{2}, \bar{C}, E_{6}, E_{5}, E_{4}$, and let $F$ be the $\pi$-exceptional curve such that $\pi(F)$ is a point of type $\mathbb{A}_{1}$. Then

$$
\beta\left(E_{2}\right) \cdot \beta\left(E_{2}\right)=\beta\left(E_{3}\right) \cdot \beta\left(E_{3}\right)=\beta(F) \cdot \beta(F)=-1,
$$


and $\check{X}$ is a smooth del Pezzo surface such that $K_{\check{X}}^{2}=6$. Thus, there exists an irreducible smooth rational curve $\check{L}_{3}$ on the surface $\check{X}$ such that $\check{L}_{3} \cdot \check{L}_{3}=-1, \check{L}_{3} \cdot \beta\left(E_{3}\right)=1$ and $\check{L}_{3} \cdot \beta(F)=0$.

Let $\bar{L}_{3}$ be the proper transform of the curve $\check{L}_{3}$ on the surface $\bar{X}$. Then $\bar{L}_{3} \cdot \bar{L}_{3}=-1$ and

$$
-K_{\bar{X}} \cdot \bar{L}_{3}=E_{3} \cdot \bar{L}_{3}=1 \text {, }
$$

which implies that $E_{1} \cdot \bar{L}_{3}=E_{2} \cdot \bar{L}_{3}=E_{4} \cdot \bar{L}_{3}=E_{5} \cdot \bar{L}_{3}=E_{6} \cdot \bar{L}_{3}=\bar{C} \cdot \bar{L}_{3}=F \cdot \bar{L}_{3}=0$.

Put $\bar{L}_{4}=\tau\left(\bar{L}_{3}\right)$ and $\bar{L}_{5}=\tau\left(\bar{L}_{2}\right)$. Then $\bar{C} \cdot \bar{L}_{4}=\bar{C} \cdot \bar{L}_{5}=0$ and

$$
-K_{\bar{X}} \cdot \bar{L}_{4}=-K_{\bar{X}} \cdot \bar{L}_{5}=E_{4} \cdot \bar{L}_{4}=E_{5} \cdot \bar{L}_{5}=1
$$

which implies that $E_{i} \cdot \bar{L}_{5}=E_{j} \cdot \bar{L}_{4}=0$ for every $i \neq 5$ and $j \neq 4$.

Put $L_{3}=\pi\left(\bar{L}_{3}\right), L_{4}=\pi\left(\bar{L}_{4}\right), L_{2}=\pi\left(\bar{L}_{2}\right)$ and $L_{5}=\pi\left(\bar{L}_{5}\right)$. Then

$$
L_{3}+L_{4} \sim L_{2}+L_{5} \sim-2 K_{X}
$$

which implies that $\mathrm{c}\left(X, L_{3}+L_{4}\right)=1 / 3$ and $\mathrm{c}\left(X, L_{2}+L_{5}\right)=1 / 2$. Then $\operatorname{lct}_{2}(X) \leqslant 2 / 3$. But

$$
\begin{gathered}
\bar{L}_{2} \sim_{\mathbb{Q}} \pi^{*}\left(L_{2}\right)-\frac{5}{7} E_{1}-\frac{10}{7} E_{2}-\frac{8}{7} E_{3}-\frac{6}{7} E_{4}-\frac{4}{7} E_{5}-\frac{2}{7} E_{6}-\frac{1}{2} F, \\
\bar{L}_{3} \sim_{\mathbb{Q}} \pi^{*}\left(L_{3}\right)-\frac{4}{7} E_{1}-\frac{8}{7} E_{2}-\frac{12}{7} E_{3}-\frac{9}{7} E_{4}-\frac{6}{7} E_{5}-\frac{3}{7} E_{6},
\end{gathered}
$$

which implies that $\mathrm{c}\left(X, 2 L_{2}+L_{3}\right)=1 / 4$. Then $2 L_{2}+L_{3} \sim_{\mathbb{Q}}-3 K_{X}$, since $\operatorname{Pic}(X) \cong \mathbb{Z}^{2}$ and

$$
L_{2} \cdot L_{2}=\frac{3}{7}, L_{3} \cdot L_{3}=\frac{5}{7}, L_{2} \cdot L_{3}=\frac{8}{7},
$$

but $2 L_{2}+L_{3}$ is a Cartier divisor, which implies that $2 L_{2}+L_{3} \sim-3 K_{X}$.

If $D$ is not a curve in $\left|-K_{X}\right|$ and $D \neq\left(L_{3}+L_{4}\right) / 2$, then arguing as in the proof of Lemma4.11, we easily see that $\mu>2 / 3$, since we can use (6.4). The lemma is proved (see Example 1.27).

Lemma 6.8. Suppose that $m=5$. Then $\mu \geqslant \operatorname{lct}_{2}(X)=2 / 3$, and if $\mu=2 / 3$, then

- either $D$ is a curve in $\left|-K_{X}\right|$ with a cusp at a point in $\operatorname{Sing}(X)$ of type $\mathbb{A}_{2}$,

- or the divisor $D$ is uniquely defined and can be explicitly described.

Proof. The curve $R^{\prime}$ has an ordinary tacnodal singularity at the point $\omega^{\prime}\left(P^{\prime}\right)$, which implies that there exists a line $L^{\prime} \subset \mathbb{P}^{2}$ such that either $L^{\prime} \subset \operatorname{Supp}\left(R^{\prime}\right)$ or $L^{\prime} \not \subset \operatorname{Supp}\left(R^{\prime}\right)$ and

$$
\operatorname{mult}_{\omega^{\prime}\left(P^{\prime}\right)}\left(L^{\prime} \cdot R^{\prime}\right)=4 \text {. }
$$

There are irreducible smooth rational curves $L_{3}^{\prime}$ and $L_{4}^{\prime}$ on the surface $X^{\prime}$ such that

$$
\omega^{\prime}\left(L_{3}^{\prime}\right)=\omega^{\prime}\left(L_{4}^{\prime}\right)=L^{\prime}
$$

and $L_{3}^{\prime}=L_{4}^{\prime} \Longleftrightarrow L^{\prime} \subset \operatorname{Supp}\left(R^{\prime}\right)$. Note that neither $L_{3}^{\prime}$ nor $L_{4}^{\prime}$ contains a point in $\operatorname{Sing}\left(X^{\prime}\right) \backslash R^{\prime}$.

Let $\bar{L}_{3}^{\prime}$ be the proper transform of the curve $L_{3}^{\prime}$ on the surface $\bar{X}^{\prime}$. Then

$$
\bar{L}_{3}^{\prime} \cap \eta\left(E_{1}\right)=\bar{L}_{3}^{\prime} \cap \eta\left(E_{2}\right)=\bar{L}_{3}^{\prime} \cap \eta\left(E_{4}\right)=\bar{L}_{3}^{\prime} \cap \eta\left(E_{5}\right)=\varnothing,
$$

and $\bar{L}_{3}^{\prime} \cdot \eta\left(E_{3}\right)=1$. Let $\bar{L}_{4}^{\prime}$ be the proper transform of the curve $L_{4}^{\prime}$ on the surface $\bar{X}^{\prime}$. Then

$$
\bar{L}_{4}^{\prime} \cap \eta\left(E_{1}\right)=\bar{L}_{4}^{\prime} \cap \eta\left(E_{2}\right)=\bar{L}_{4}^{\prime} \cap \eta\left(E_{4}\right)=\bar{L}_{4}^{\prime} \cap \eta\left(E_{5}\right)=\varnothing,
$$

and $\bar{L}_{4}^{\prime} \cdot \eta\left(E_{3}\right)=1$. One can also check that $\bar{L}_{3}^{\prime} \cap \bar{L}_{4}^{\prime}=\varnothing$ if $\bar{L}_{3}^{\prime} \neq \bar{L}_{4}^{\prime}$.

Let $\bar{L}_{3}$ and $\bar{L}_{4}$ be the proper transforms of the curves $\bar{L}_{3}^{\prime}$ and $\bar{L}_{4}^{\prime}$ on the surface $\bar{X}$, respectively, and let us put $L_{3}=\pi\left(\bar{L}_{3}\right)$ and $L_{4}=\pi\left(\bar{L}_{4}\right)$. Then

$$
\bar{L}_{3}+\bar{L}_{4} \sim-2 K_{X}
$$

and $\mathrm{c}\left(X, \bar{L}_{3}+\bar{L}_{4}\right)=1 / 3$, which implies that $\operatorname{lct}_{2}(X) \leqslant 2 / 3$.

If $D \neq\left(\bar{L}_{3}+\bar{L}_{4}\right) / 2$, then (6.4), the proof of Lemma 4.10 and Lemma 2.6 imply that

$$
\mu \geqslant \operatorname{lct}_{2}(X)=\frac{2}{3} \text {. }
$$


and if $\mu=2 / 3$, then $D$ is a curve in $\left|-K_{X}\right|$ with a cusp at a point in $\operatorname{Sing}(X)$ of type $\mathbb{A}_{2}$.

Lemma 6.9. Suppose that $m=4$. Then

$$
\mu \geqslant \operatorname{lct}_{2}(X)=\min \left(\operatorname{lct}_{1}(X), 4 / 5\right) \geqslant \frac{2}{3},
$$

and if $\mu=2 / 3$, then $D$ is a curve in $\left|-K_{X}\right|$ with a cusp at a point in $\operatorname{Sing}(X)$ of type $\mathbb{A}_{2}$.

Proof. The point $\omega^{\prime}\left(P^{\prime}\right)$ is an ordinary cusp of the curve $R^{\prime}$. Then there is a line $L^{\prime} \subset \mathbb{P}^{2}$ such that

$$
\operatorname{mult}_{\omega^{\prime}\left(P^{\prime}\right)}\left(L^{\prime} \cdot R^{\prime}\right)=3
$$

Let $Z^{\prime}$ be a curve in $X^{\prime}$ such that $\omega^{\prime}\left(Z^{\prime}\right)=L^{\prime}$ and $-K_{X^{\prime}} \cdot Z^{\prime}=2$. Then

$$
Z^{\prime} \cap \operatorname{Sing}\left(X^{\prime}\right)=\operatorname{Sing}\left(Z^{\prime}\right)=R^{\prime},
$$

the $Z^{\prime}$ is irreducible curve that has an ordinary cusp at the point $R^{\prime}$.

Let $\bar{Z}^{\prime}$ be the proper transform of the curve $Z^{\prime}$ on the surface $\bar{X}^{\prime}$. Then $Z^{\prime}$ is smooth and

$$
\eta\left(E_{2}\right) \cap \eta\left(E_{3}\right) \in \bar{Z}^{\prime} .
$$

Let $\bar{Z}$ be the proper transform of the curve $\bar{Z}^{\prime}$ on the surface $\bar{X}$. Put $Z=\pi(\bar{Z})$. Then

$$
\bar{Z} \sim \pi^{*}(Z)-E_{1}-2 E_{2}-2 E_{3}-E_{4}
$$

and $E_{2} \cap E_{3} \in Z$. Then $\mathrm{c}(X, Z)=2 / 5$, which implies that $\operatorname{lct}_{2}(X) \leqslant 4 / 5$.

Arguing as in the proof of Lemma 4.8 and using Lemma 2.6 and (6.4), we see that

$$
\mu \geqslant \operatorname{lct}_{2}(X)=\min \left(\operatorname{lct}_{1}(X), 4 / 5\right)
$$

and if $\mu=2 / 3$, then $D$ is a curve in $\left|-K_{X}\right|$ with a cusp at a point in $\operatorname{Sing}(X)$ of type $\mathbb{A}_{2}$.

The assertion of Theorem 6.1 is proved.

\section{REFERENCES}

[1] V. Alexeev, V. Nikulin, Del Pezzo and K3 Surfaces

MSJ Memoirs 15 (2006), Mathematical Society of Japan, Tokyo

[2] I. Cheltsov, Log canonical thresholds on hypersurfaces Sbornik: Mathematics 192 (2001), 1241-1257

[3] I. Cheltsov, Log canonical thresholds of del Pezzo surfaces Geometric and Functional Analysis, 18 (2008), 1118-1144

[4] I. Cheltsov, On singular cubic surfaces Asian Journal of Mathematics 19 (2009), 191-214

[5] I. Cheltsov, J. Park, J. Won, Log canonical thresholds of certain Fano hypersurfaces arXiv:0706.0751 (2007)

[6] I. Cheltsov, C. Shramov, Log canonical thresholds of smooth Fano threefolds Russian Mathematical Surveys 63 (2008), 859-958

[7] I. Cheltsov, C. Shramov, On exceptional quotient singularities Geometry and Topology 15 (2011), 1843-1882

[8] X. Chen, B. Wang, Kähler-Ricci flow on Fano manifolds arXiv:0909.2391 (2009)

[9] F. Cools, M. Coppens, Star points on smooth hypersurfaces Journal of Algebra 323 (2010), 261-286

[10] J.-P. Demailly, J. Kollár, Semi-continuity of complex singularity exponents and Kähler-Einstein metrics on Fano orbifolds

Annales Scientifiques de l'École Normale Supérieure 34 (2001), 525-556

[11] W. Ding, G. Tian, Kähler-Einstein metrics and the generalized Futaki invariant Inventiones Mathematicae, 110 (1992), 315-335 
[12] S. Donaldson, Stability, birational transformations and the Kahler-Einstein problem arXiv:1007.4220 (2010)

[13] T. de Fernex, L. Ein, M. Mustata, Bounds for log canonical thresholds with applications to birational rigidity Mathematical Research Letters 10 (2003), 219-236

[14] A. Futaki, An obstruction to the existence of Einstein-Kähler metrics Inventiones Mathematicae 73 (1983), 437-443

[15] J. Gauntlett, D. Martelli, J. Sparks, S.-T. Yau, Obstructions to the existence of Sasaki-Einstein metrics Communications in Mathematical Physics 273 (2007) 803-827

[16] A. Ghigi, J. Kollár,Kähler-Einstein metrics on orbifolds and Einstein metrics on spheres Commentarii Mathematici Helvetici, 82 (2007), 877-902

[17] P. Hacking, Y. Prokhorov, Smoothable del Pezzo surfaces with quotient singularities Compositio Mathematica 146 (2010), 169-192

[18] F. Hidaka, K. Watanabe, Normal Gorenstein surfaces with ample anti-canonical divisor Tokyo Journal of Mathematics 4 (1981), 319-330

[19] T. Jeffres, Singular set of some Kähler orbifolds Transactions of the American Mathematical Society 349 (1997), 1961-1971

[20] S. Keel, J. McKernan, Rational curves on quasi-projective surfaces Memoirs of the American Mathematical Society 669 (1999), American Mathematical Society

[21] J. Kollár, Singularities of pairs Proceedings of Symposia in Pure Mathematics 62 (1997), 221-287

[22] J. Kollár et al., Flips and abundance for algebraic threefolds Astérisque 211 (1992)

[23] G. Lyubeznik, On Bernstein-Sato polynomials Proceedings of the American Mathematical Society 125 (1997), 1941-1944

[24] T. Mabuchi, S. Mukai, Stability and Einstein-Kähler metric of a quartic del Pezzo surface Lecture Notes in Pure and Applied Mathematics 145 (1993), 133-160

[25] Y. Matsushima, Sur la structure du groupe d'homéomorphismes analytiques d'une certaine variété kählérienne Nagoya Mathematical Journal 11 (1957), 145-150

[26] M. Miyanishi, D.-Q. Zhang, Gorenstein log del Pezzo surfaces of rank one Journal of Algebra 118 (1988), 63-84

[27] M. Mustata, Singularities of pairs via jet schemes Journal of the American Mathematical Society 15 (2002), 599-615

[28] J. Park, Birational maps of Del Pezzo fibrations Journal fur die Reine und Angewandte Mathematik 538 (2001), 213-221

[29] J. Park, A note on del Pezzo fibrations of degree 1 Communications in Algebra, 31 (2003), 5755-5768

[30] J. Park, J. Won Log canonical thresholds on Gorenstein canonical del Pezzo surfaces Proceedings of the Edinburgh Mathematical Society, 54 (2011), 187-219

[31] J. Park, J. Won Log canonical thresholds on del Pezzo surfaces of degree $\geqslant 2$ Nagoya Mathematical Journal, 200 (2010), 1-26

[32] Yu.Prokhorov, Blow-ups of canonical singularities arXiv:math/9810097 (1998)

[33] A. Pukhlikov, Birational geometry of Fano direct products Izvestiya: Mathematics 69 (2005), 1225-1255

[34] J. Ross, R. Thomas, Weighted projective embeddings, stability of orbifolds and constant scalar curvature Kahler metrics arXiv:math/09075214 (2009)

[35] Y.Shi, On the $\alpha$-invariants of cubic surfaces with Eckardt points arXiv:0902.3203 (2009)

[36] G. Tian, On Kähler-Einstein metrics on certain Kähler manifolds with $c_{1}(M)>0$ Inventiones Mathematicae 89 (1987), 225-246 
[37] G. Tian, On Calabi's conjecture for complex surfaces with positive first Chern class Inventiones Mathematicae, 101 (1990), 101-172

[38] G. Tian, On a set of polarized Kähler metrics on algebraic manifolds Journal of Differential Geometry 32 (1990), 99-130

[39] G. Tian, Kähler-Einstein metrics with positive scalar curvature Inventiones Mathematicae 130 (1997), 1-37

[40] T. Urabe, On singularities on degenerate Del Pezzo surfaces of degree 1, 2 Proceedings of Symposia in Pure Mathematics 40 (1983), 587-590

[41] B. Wang, Ricci flow on orbifolds arXiv:1003.0151 (2010)

School of Mathematics, University of Edinburgh, Edinburgh EH9 3JZ, UK I.Cheltsov@ed. AC. UK

DIMITRAKOSTA@HOTMAIL. COM 